\title{
Fluctuations in cool quark matter and the phase diagram of quantum chromodynamics
}

\author{
Robert D. Pisarski, ${ }^{1}$ Vladimir V. Skokov, ${ }^{2}$ and Alexei M. Tsvelik ${ }^{3}$ \\ ${ }^{1}$ Department of Physics, Brookhaven National Laboratory, Upton, New York 11973, USA \\ ${ }^{2}$ RIKEN/BNL Research Center, Brookhaven National Laboratory, Upton, New York 11973, USA \\ ${ }^{3}$ Condensed Matter Physics and Materials Science Division, Brookhaven National Laboratory, \\ Upton, New York 11973-5000, USA
}

(Received 6 March 2018; revised manuscript received 10 December 2018; published 29 April 2019)

\begin{abstract}
We consider the phase diagram of hadronic matter as a function of temperature, $T$, and baryon chemical potential, $\mu$. Currently the dominant paradigm is a line of first order transitions which ends at a critical endpoint. In this work we suggest that spatially inhomogeneous phases are a generic feature of the hadronic phase diagram at nonzero $\mu$ and low $T$. Familiar examples are pion and kaon condensates. At higher densities, we argue that these condensates connect onto chiral spirals in a quarkyonic regime. Both of these phases exhibit the spontaneous breaking of a global $U(1)$ symmetry and quasilong range order, analogous to smectic liquid crystals. We argue that there is a continuous line of first order transitions which separate spatially inhomogeneous from homogeneous phases, where the latter can be either a hadronic phase or a quark-gluon plasma. While mean field theory predicts that there is a Lifshitz point along this line of first order transitions, in three spatial dimensions strong infrared fluctuations wash out any Lifshitz point. Using known results from inhomogeneous polymers, we suggest that instead there is a Lifshitz regime. Nonperturbative effects are large in this regime, where the momentum dependent terms for the propagators of pions and associated modes are dominated not by terms quadratic in momenta, but quartic. Fluctuations in a Lifshitz regime may be directly relevant to the collisions of heavy ions at (relatively) low energies, $\sqrt{s} / A: 1 \rightarrow 20 \mathrm{GeV}$.
\end{abstract}

DOI: 10.1103/PhysRevD.99.074025

\section{INTRODUCTION}

The phases of quantum chromodynamics (QCD), as a function of temperature, $T$, and the baryon (or equivalently, quark) chemical potential, $\mu$, are of fundamental interest [1]. At zero chemical potential, numerical simulations on the lattice indicate that there is no true phase transition, just a crossover, albeit one where the degrees of freedom increase dramatically [2-4]. At a nonzero chemical potential, however, a crossover line may meet a line of first order transitions at a critical endpoint [5-10]. These lines separate two phases: a hadronic phase in which chiral symmetry is spontaneously broken, and a (nearly) chirally symmetric phase of quarks and gluons.

In condensed matter it is well known that a third phase can arise, in which spatially inhomogeneous structures form. If so, the three phases meet at a Lifshitz point [11-25].

Published by the American Physical Society under the terms of the Creative Commons Attribution 4.0 International license. Further distribution of this work must maintain attribution to the author(s) and the published article's title, journal citation, and DOI. Funded by SCOAP ${ }^{3}$.
In hadronic nuclear matter the existence of spatially inhomogeneous phases is familiar, as pionic [26-39] and kaonic [40-43] condensates. They are ubiquitous in GrossNeveu models in $1+1$ dimensions, which are soluble either for a large number of flavors [44-49] or by using advanced nonperturbative techniques [50]. These phases also arise from analyses of effective models of QCD, where they have been termed chiral spirals [51-106]. In this paper we consider especially the role played by fluctuations in phases with spatially inhomogeneous phases, and show that they can dramatically affect the phase diagram of QCD.

At densities between a dense hadronic phase and deconfined quarks, cool quark matter is quarkyonic: while the pressure is (approximately) perturbative, the excitations near the Fermi surface are confined [102]. The Fermi surface of quarks, which starts out as isotropic, breaks up into a set of patches, with the longitudinal fluctuations governed by a Wess-Zumino-Novikov-Witten Lagrangian [103-105]. The transverse fluctuations, however, are of higher order in momenta: they are not quadratic, but quartic. Among condensed matter systems the closest analogy are smectic liquid crystals, which consist of elongated molecules periodically ordered in just one direction [13]. The color and flavor quantum numbers which quarks carry makes the 
analogous state more involved. As for pion/kaon condensates [34-39,97-100], at nonzero temperature there are long range correlations in the inhomogeneous phase, and there is no true order parameter. At nonzero temperature in $3+1$ dimensions, the fluctuations exhibit complicated patterns. The propagators of two-quark operators decay exponentially with a temperature dependent correlation length, and propagators of spin and flavor singlet operators, composed of $2 N_{f}$ quarks, fall off as a power law.

We then consider how a quarkyonic phase matches onto the usual hadronic phase at low temperature and density. We first discuss how as the density decreases, the chiral spirals in a quarkyonic phase transform naturally into the pion/kaon condensates of hadronic nuclear matter. We show that the phase diagram valid in mean field theory - two lines of second order phase transitions, meeting a line of first order transitions at a Lifshitz point-is dramatically altered by fluctuations. As demonstrated first by Brazovskii [89,107-109], the line of transitions between the symmetric phase and that with chiral spirals becomes a line of first order transitions. Most importantly, the infrared fluctuations about a Lifshitz point are so strong that there is, in fact, no true Lifshitz point [15-17,23-25]. This is known to occur in inhomogeneous polymers, both from experiment and numerical simulations [18-22]. We suggest that in QCD, infrared fluctuations also wipe out the Lifshitz point, leaving just a line of first order transitions separating the region with inhomogeneous phases from those without. What remains is a Lifshitz regime, which is illustrated in Figs. 2 and 3 below. The Lifshitz regime is manifestly nonperturbative, as the momentum dependence for the propagators for pions (or the associated modes of a chiral spiral) are dominated by terms quartic, instead of quadratic, in the momenta. This change in the momentum dependence generates large fluctuations, which may be related to known [106,110-115] and possible [116,117] anomalies in the collisions of heavy ions at center of mass energies per nucleon $\sqrt{s} / A: 1 \rightarrow 20 \mathrm{GeV}$.

\section{THE MODEL OF QUARKYONIC PHASE}

A quarkyonic phase only exists when quark excitations near the Fermi surface are (effectively) confined. While for three colors numerical simulations of lattice gauge theories at nonzero quark density are afflicted by the sign problem for three colors, they are possible for two colors [118-123]. Although the original argument for a quarkyonic phase was based upon the limit of a large number of colors [102], these simulations show that even for two colors, the expectation value of the Polyakov loop is small, indicating confinement, up to large values of the quark chemical potential [118-123]. Directly relevant to our analysis are the results of Bornyakov et al., who find that the string tension decreases gradually from its value in vacuum, to essentially zero at $\mu_{q} \sim 750 \mathrm{MeV}$ : see Fig. 3 of Ref. [123]. This suggests that a quarkyonic phase may dominate for a wide range of chemical potential, and indeed, for all values relevant to hadronic stars $[124,125]$.

There is an elementary argument for why there could be such a large region in which cool quark matter is quarkyonic. Consider computing a scattering process in vacuum. By asymptotic freedom, this is certainly valid at large momentum. As the momentum decreases, nonperturbative effects enter, and a perturbative computation is invalid. This certainly occurs by momenta $\Lambda_{\text {scat }} \sim 1 \mathrm{GeV}$, if not before.

Now consider computing the pressure perturbatively. The scattering processes which contribute involve the scattering of quarks and holes with momenta whose magnitude is on the order of the Fermi momentum. This suggests that the pressure can be computed perturbatively down to a scale which is typical of that where perturbative computations are valid: that is, on the order of $\Lambda_{\text {scat }} \sim 1 \mathrm{GeV}$. This argument is clearly qualitative: the estimate of $\Lambda_{\text {scat }}$ as applying to the perturbative computation of the pressure could well vary by a factor of two. Furthermore, our argument applies only to the pressure: excitations about the Fermi surface involve much smaller energies than the chemical potential, so that one expects a transition from the perturbative, to the quarkyonic, regime [102]. What is important is that the momentum scale $\Lambda_{\text {scat }}$ does not depend strongly upon the number of colors. This elementary argument may explain why there is a large quarkyonic regime even for two colors [118-123].

This estimate differs from that at zero quark density and a nonzero temperature, $T$. In computing the pressure at $T \neq 0$ and $\mu_{q}=0$, the dominant momentum scale is naively the first Matsubara frequency, $=2 \pi T$ [126,127]. Detailed computations to two loop order $[128,129]$ show that the precise value is a bit larger, but this estimate is qualitatively correct. At temperatures $\sim 150 \mathrm{MeV}$, this is about $\sim 1 \mathrm{GeV}$, which is the same as we estimate for $T=0$ and $\mu_{q} \neq 0$.

We note that a quantitative measure of what momentum scale perturbative computations of the pressure are valid will be provided by computations to high order, such as $\sim g^{6}$, as are currently underway $[130,131]$.

Returning to the quarkyonic phase, previously we assumed that the chiral symmetry was restored [103-105]. However, for the reasons of convenience we will work with the nonrelativistic limit of the quark Hamiltonian which is formally justified if the constituent quark mass is nonzero. At nonzero quark mass, we work with the nonrelativistic limit of the quark Hamiltonian:

$$
\begin{aligned}
H= & \int d^{3} x\left\{\psi _ { \alpha , f , a } ^ { + } \left[\frac{1}{2 m_{f}}\left(\mathrm{i} \boldsymbol{\nabla}-\frac{q_{f}}{c} \mathbf{A}\right)^{2}\right.\right. \\
& +\left(q_{f} / m_{f} c^{2}\right)(\boldsymbol{\sigma}[\boldsymbol{\nabla} \times \mathbf{A}]) \\
& \left.\left.-\left(\mu-m_{f} c^{2}\right)\right] \psi_{\alpha, f, a}+V_{1}+V_{2}\right\}
\end{aligned}
$$




$$
\begin{aligned}
& V_{1}=\frac{1}{2}\left(\psi^{+}(r) T^{A} \psi(r)\right) D_{00}\left(\left|\mathbf{r}-\mathbf{r}^{\prime}\right|\right)\left(\psi^{+}\left(r^{\prime}\right) T^{A} \psi\left(r^{\prime}\right)\right), \\
& V_{2}=\frac{1}{2}\left(\psi^{+}(r) \boldsymbol{\sigma} \otimes T^{A} \psi(r)\right) D_{\perp}\left(r-r^{\prime}\right)\left(\psi^{+}\left(r^{\prime}\right) \boldsymbol{\sigma} \otimes T^{A} \psi\left(r^{\prime}\right)\right),
\end{aligned}
$$

where $\psi^{+} T^{A} \psi=\left(\psi_{\alpha, f ; a}^{+}(r) T_{a b}^{A} \psi_{\alpha, f ; b}(r)\right)$ and $\psi^{+} \otimes \sigma^{a} T^{A} \psi=$ $\left(\psi_{\alpha, f ; a}^{+}(r) \sigma_{\alpha \beta}^{a} T_{a b}^{A} \psi_{\beta, f ; b}(r)\right)$. We assume that there is single gluon exchange, where $D_{00}$ is a confining propagator,

$$
D_{00}(\mathbf{p})=\frac{\sigma_{0}}{\left(|\mathbf{p}|^{2}\right)^{2}},
$$

and $D_{\perp}$ is perturbative,

$$
D_{\perp}(p)=\frac{g^{2}}{p^{2}} .
$$

Near the Fermi surface antiquarks can be ignored, with $\psi_{\alpha, f, n}^{+}$ and $\psi_{\alpha, f, n}$ being creation and annihilation operators of nonrelativistic fermions carrying spin (not Dirac spinors) about the Fermi surface. Here $\alpha= \pm 1 / 2$ is a spin projection, $a, b \ldots=1,2,3$ color indices, and $f=1, \ldots N_{f}$ the flavor index. ( $N_{f}=2$ for up and down quarks, $N_{f}=3$ if we include the strange quark) $q_{f}, m_{f}$ are electric charges and masses of the quarks, and $\mathbf{A}$ is an external vector potential for QED. The operators $T^{A}$ are generators of color $S U(3)$ group, $\sigma^{a}$ are Pauli matrices.

In the first approximation we neglect the asymmetry introduced by differences in $q_{f}$ and $m_{f}$ and by the magnetic interaction $V_{2}$. Then we can treat $j=(\alpha, f)$ as a united set of indices, so the theory (1) is invariant under a larger symmetry of $S U\left(2 N_{f}\right)$. Then realistic values are $2 N_{f}=4$ (low density, no strange quarks) or $2 N_{f}=6$ for high density.

If the chiral symmetry is broken, $m_{f}$ are renormalized quark masses. In fact, exact definitions of $m_{f}$ do not affect the qualitative side of our arguments since for excitations near the Fermi surface the only difference between massless and massive quarks is the difference in the Fermi velocity, $v_{F}$, which follows from the relationship between energy and momentum. We also neglect the frequency dependence of the gluon propagator $D_{00}$, and set $c=1$.

The extended symmetry of $S U\left(2 N_{f}\right)$, instead of $S U\left(N_{f}\right)$, is due to a doubling from the spin degrees of freedom, and is no longer respected once magnetic interactions (3) are included. The same symmetry has been discussed, both in the hadronic spectrum and at nonzero quark density, in Refs. [132-135].

As was demonstrated previously [104] the quarkyonic phase supports a collection of spin-flavor density waves. Since in the nonrelativistic limit spin and flavor are treated on equal footing, a wave with wave vector $\mathbf{Q}$ is characterized by the slow order parameter field in the form of a $2 N_{f} \times 2 N_{f}$ matrix field $U_{Q}$ such that at long distances the spin-flavor density can be decomposed as

$\rho_{j k}=\sum_{n=1}^{N_{c}} \psi_{j, n}^{+}(\mathbf{r}) \psi_{k, n}(\mathbf{r})-\rho_{0} \delta_{j k}=\sum_{\mathbf{Q}} U_{Q}^{j k}(\mathbf{r}) \mathrm{e}^{\mathrm{i} \mathbf{Q r}}$,

where $\rho_{0}$ is the average density. The set of wave vectors $\mathbf{Q}_{i}$ and amplitudes of the matrix fields related to $\operatorname{det} U$ are determined by the matter density, which follows from the value of the chemical potential. In condensed matter systems a density wave with wave vector $\mathbf{Q}$ usually forms when the parts of the Fermi surface connected by $\mathbf{Q}$ can be superimposed on each other, which is the nesting condition. Once this condition is fulfilled, the susceptibility acquires a singularity at $\mathbf{Q}$ signifying a possible instability. However, for cold quarks a perfect nesting is unnecessary due to the singular character of the confining potential in Eq. (2) [104]. As a result quarks can scatter with one another only at small angles and still remain near the edge of the Fermi surface. So it is sufficient to fulfill the nesting condition just on limited patches of the Fermi surface whose area, $\Lambda^{2}$, is determined by the interplay between the string tension $\sigma$ and the curvature of the Fermi surface. Within these patches the problem is essentially one dimensional, and can be treated by non-Abelian bosonization and conformal embedding $[50,136]$.

In the first approximation when we neglect the magnetic interaction (3) and maintain the $S U\left(2 N_{f}\right)$ symmetry of the Hamiltonian the conformal embedding works as follows. In non-Abelian bosonization the noninteracting one dimensional Hamiltonian can be written as a sum of WessZumino-Novikov-Witten (WZNW) terms of $U(1)$ for charge, $S U_{3}\left(2 N_{f}\right)$ for spin and flavor, and $S U_{2 N_{f}}(3)$ for color $[103-105,136]$. The decomposition is adjusted to the symmetry of the interaction (2) which is given by the product of the $S U_{2 N_{f}}(3)$ Kac-Moody currents which commute with the first two WZNW Hamiltonians. As a result only the color sector experiences confinement and the other two remain massless. They represent Abelian and non-Abelian Goldstone modes, and so the corresponding correlators have power law fall off at large distances.

We show that due to the arbitrariness of the choice of direction of the Q's, the modes which have a linear spectrum in one dimension acquire a quadratic dispersion in the direction along the Fermi surface when transverse derivatives are included. This coupling together with the magnetic interaction (3) also breaks the extended symmetry from $S U\left(2 N_{f}\right)$ down to $S U\left(N_{f}\right)$.

The minimal possible number of patches is six, as a cube embedded into a spherical Fermi surface. When the density increases it becomes energetically advantageous to form triangular patches at the corners of the cube, so another eight patches, with fourteen in all. 
We estimate the number of patches as follows. The effective current-current interaction in (1) scales to strong coupling giving rise to a characteristic energy scale, $\Delta$. This can be estimated from the self-consistency condition in the rainbow diagram with one gluon and one quark propagator:

$$
\begin{aligned}
\Delta(\mathbf{Q})= & \int \frac{d \omega d^{3} q}{(2 \pi)^{4}} D_{00}(\mathbf{q})\langle\langle\psi(\omega, \mathbf{Q} / 2+\mathbf{q}) \\
& \left.\left.\times \psi^{+}(\omega,-\mathbf{Q} / 2+\mathbf{q})\right\rangle\right\rangle \\
\sim & \sigma_{0} \int_{\Delta} \frac{d^{2} q_{\perp} d q_{\|}}{\left(q_{\perp}^{2}+q_{\|}^{2}\right)^{2}} \int \frac{d \omega \Delta}{\omega^{2}+\left(v_{F} q_{\|}\right)^{2}} \sim \frac{\sigma_{0} v_{F}}{\Delta},
\end{aligned}
$$

where $v_{F}$ is the Fermi velocity and $|\mathbf{Q}|=2 k_{F}$. The size of the patch is estimated setting the transverse part of the quarks' kinetic energy equal to this scale:

$$
p_{\perp}^{2} / 2 m=\Delta, \quad \Lambda^{2}=\pi p_{\perp}^{2} \sim m \sqrt{v_{F} \sigma_{0}} .
$$

From (9) we find the following estimate for the number of patches:

$$
N_{\text {patches }} \sim \frac{k_{F}^{2}}{\Lambda^{2}} \sim \sqrt{\frac{n}{m \sigma_{0}}},
$$

where $n$ is the density of quarks. Since $\sigma_{0}$ is essentially zero above some value of the chemical potential, for massive quarks the number of patches first grows and than sharply decreases with density. The estimate of Eq. (10) also shows the dependence of the number of patches on the quark mass $m$. For massless quarks, $p_{\perp} \sim \Delta$, and $\Lambda^{2} \sim \sigma_{0}$. Thus chiral symmetry breaking decreases the number of patches.

The quantum action for an individual patch describes the baryonic strange metal described in Ref. [50]: the quarks are confined, but the baryons remain gapless and incoherent. The only coherent excitations are the bosonic collective modes, so that the corresponding phase can be characterized as a Bose metal.

\section{GINZBURG- LANDAU DESCRIPTION OF QUARKIONIC CRYSTAL PHASE}

In this section we return to the problem of description of the quarkyonic crystal phase. We show that at nonzero temperature there is no long range order and that this state resembles smectic liquid crystals, which are ordered periodically only in one direction, although with some significant caveats.

As we have mentioned above, the action for the fluctuations normal to the Fermi surface is given by the sum of WZNW actions for each patch. For the static components of the order parameter fields one can omit the Wess-Zumino terms, leaving just terms from the gradient expansion. The corresponding Ginzburg-Landau (GL) free energy for the fields $U$ was written in [104], but it requires correction. It is known [34-39,97-100] that the fluctuations tangential to the Fermi surface must have a zero stiffness, since the orientation of the entire set of $\mathbf{Q}_{i}{ }^{\text {'s }}$ is arbitrary and for spherical Fermi surface any such rotation costs zero energy. Therefore at nonzero temperature where all fluctuations are classical, the free energy density similar to that in smectic liquid crystal [97]:

$$
\begin{aligned}
\mathcal{F} / T= & \frac{1}{2 T} \sum_{\mathbf{q}}\left\{\tilde{\lambda}_{1, Q} \operatorname{Tr}\left(\mathbf{q} \nabla U_{Q}\right)\left(\mathbf{q} \nabla U_{Q}^{+}\right)\right. \\
& \left.\left.+\tilde{\lambda}_{2, Q} \operatorname{Tr}\left[(\mathbf{q} \times \nabla)^{2} U_{Q}\right)\right]\left[(\mathbf{q} \times \nabla)^{2} U_{Q}^{+}\right]\right\}+\mathcal{V}\left(U^{+}, U\right), \\
\mathbf{q}= & \mathbf{Q} / Q,
\end{aligned}
$$

where $U_{-\mathbf{Q}}=U_{\mathbf{Q}}^{+}$and $\mathcal{V}$ is the local potential which fixes the amplitudes of these matrix fields. We normalize the Q's as unit vectors. Equation (11) can be formally derived from Eq. (1). The first term was derived in our previous paper [104], while the second originates from the fusion of the two perturbing operators

$$
\begin{gathered}
\hat{T}_{\perp}=-\frac{v_{F}}{2 k_{F}}\left(R^{+} \nabla_{\perp}^{2} R+L^{+} \nabla_{\perp}^{2} L\right), \\
R(\mathbf{p})=\psi(\mathbf{Q} / 2+\mathbf{p}), \quad L(\mathbf{p})=\psi(-\mathbf{Q} / 2+\mathbf{p}) .
\end{gathered}
$$

Then the estimates of the parameters: $\lambda_{1} \sim v_{F} \Lambda^{2}$, $\lambda_{2} / \lambda_{1}=C k_{F}^{-2}$, where $C$ is a numerical constant and $k_{F}$ is the Fermi wave vector.

When the Fermi vectors of up, down and strange quarks are different, the GL theory should be augmented by the term

$$
\begin{aligned}
& \left(m_{s}-m_{u, d}\right) c^{2} \int d^{3} x \psi_{\sigma, f, n}^{+} \psi_{\sigma, f, n} \\
& \quad=\mathrm{i}\left(m_{s}-m_{u, d}\right) c^{2} \int d^{3} x \frac{N_{f}}{\pi} \operatorname{Tr}\left[\hat{\tau}^{3} U_{Q}(\mathbf{q} \nabla) U_{\mathbf{Q}}^{+}\right], \\
& \tau^{3}=\operatorname{diag}(-1,-1,2) \otimes \hat{I},
\end{aligned}
$$

where the first matrix in the tensor product acts in flavor space, and the second in spin space. This contribution is the non-Abelian bosonization of the above fermionic term. This extra contribution can be removed by the redefinition of the matrix field:

$$
U_{Q} \rightarrow \mathrm{e}^{\mathrm{i} \delta \mu \tau^{3}(\boldsymbol{Q} r)} U_{Q}
$$

So the shift of the Fermi level of strange quarks does not break the SU(6) symmetry, at least in the leading approximation in $\delta m$. A magnetic field does, which is taken into account later.

The matrix $U$ can be parametrized as

$$
U_{Q}=A_{Q} \mathrm{e}^{\mathrm{i} \phi_{Q}} G_{Q},
$$


where the amplitude $A_{Q}$ is fixed by the potential $\mathcal{V}(11)$, and $G_{Q}$ is a $S U\left(2 N_{f}\right)$ matrix. Omitting the massive fluctuations of the amplitude $A_{Q}$ we get from (11) the free energy density for the soft modes. It is divided into two parts, Abelian and non-Abelian sigma models:

$$
\begin{gathered}
\frac{\mathcal{F}}{T}=S_{U(1)}+S_{S U\left(2 N_{f}\right)}, \\
\left.S_{U(1)}=\frac{1}{2 T} \sum_{\mathbf{Q}}\left[\lambda_{1}\left(\mathbf{q} \cdot \nabla \phi_{Q}\right)^{2}+\lambda_{2}\left[(\mathbf{q} \times \nabla)^{2} \phi_{Q}\right)\right]^{2}\right], \\
S_{S U\left(2 N_{f}\right)}=\frac{1}{2 T} \sum_{\mathbf{Q}}\left\{\lambda_{1} \operatorname{Tr}\left(\mathbf{q} \cdot \nabla G_{Q}\right)\left(\mathbf{q} \cdot \nabla G_{Q}^{+}\right)\right. \\
\left.\left.+\lambda_{2} \operatorname{Tr}\left[(\mathbf{q} \times \nabla)^{2} G_{Q}\right)\right]\left[(\mathbf{q} \times \nabla)^{2} G_{Q}^{+}\right]\right\} .
\end{gathered}
$$

\section{FLUCTUATIONS AND ORDER}

We next show that because the transverse stiffness vanishes, no symmetry is broken at nonzero temperature. In that respect the quarkyonic phase resembles the lamellar phases of liquid crystals, which have the same bare fluctuation spectrum.

Under renormalization, the Abelian, Eq. (18), and nonAbelian, Eq. (19), parts of the free energy behave very differently. The Abelian part is just a free theory, since the effects of vortices in three dimensions can be ignored. On the other hand, due to the softness of the transverse fluctuations, the action of Eq. (19) renormalizes to strong coupling, which generates a finite correlation length. In the one loop approximation the renormalization group equations do not differ from those for a two-dimensional $S U\left(2 N_{f}\right)$-symmetric principal chiral field model. The analogy becomes clearer when one uses the saddle point approximation. A rough estimate for the correlation length can be obtained if we replace the local constraint for the matrix field $G G^{+}=I$ by its average $\left\langle G G^{+}\right\rangle=1$. We enforce the constraint $G G^{+}=I$ by adding to the action the term $i \eta\left(G^{+} G-I\right)$, where $\eta$ is the Lagrange multiplier field. In the saddle point approximation we replace the multiplier field $\eta$ by a constant $i \eta=\lambda_{1} \xi^{-2}$. Then we extract the propagator of $G$ from Eq. (19), as the constraint $\left\langle G G^{+}\right\rangle=1$ yields the equation for the inverse correlation length $\xi^{-1}$ :

$$
1=\frac{4 N_{f} T}{(2 \pi)^{3}} \int \frac{d k_{\|} d^{2} k_{\perp}}{\lambda_{1} k_{\|}^{2}+\lambda_{2} k_{\perp}^{4}+\lambda_{1} \xi^{-2}},
$$

where the correlation length $\xi$ is

$$
\xi \sim v_{F} \Lambda^{-1} \exp \left(\pi \sqrt{\lambda_{1} \lambda_{2}} / N_{f} T\right)=v_{F} \Lambda^{-1} \exp \left(C \frac{\Lambda^{2}}{m N_{f} T}\right)
$$

where $C \sim 1$. Thus the non-Abelian sector is disordered, although at low temperature, the correlation length is exponentially large. The Abelian action in Eq. (18) is a free theory. The corresponding observables are complex exponents of $\phi_{Q}$ and as such are periodic functionals of $\phi_{Q}$. In two spatial dimensions vortices of the $\phi_{Q}$ field would also enter, but in three spatial dimensions these vortices are extended objects with an energy proportional to their length, and so can be ignored. Thus at nonzero temperature, there is a phase transition of second order into a phase characterized by long range correlations of the fields $\phi_{Q}$.

An order parameter can be constructed for this critical phase. It cannot directly involve correlations of $\rho_{j k}$, because the correlations of such fields decay exponentially. However, since the charge phase $\phi_{Q}$ is a free field over large distances, we can construct an operator which exhibits quasi long range order. Since $\operatorname{det} G=1$ the order parameter includes $4 N_{f}$ fermions:

$$
\mathcal{O}_{\mathbf{Q}}=\operatorname{det} \hat{\rho}_{\mathbf{Q}}=\mathrm{e}^{2 \mathrm{i} N_{f}\left(\mathbf{Q r}+\phi_{Q}\right)} B_{\mathbf{Q}}
$$

At distances $\gg \xi$ when fluctuations of $G$ can be treated as massive one can replace $B$ by some fixed amplitude. The average $\left\langle\mathcal{O}_{Q}\right\rangle=0$, but its correlations fall off as powers of the relative distance:

$\left\langle\left\langle\mathcal{O}_{\mathbf{Q}}\left(\mathbf{r}_{1}\right) \mathcal{O}_{\mathbf{Q}}^{+}\left(\mathbf{r}_{2}\right)\right\rangle \sim \frac{\cos \left[2 N_{f} \mathbf{Q} \mathbf{r}_{12}\right]}{\left\{\left(\mathbf{q} \mathbf{r}_{12}\right)^{2}+k_{0}^{2}\left[\mathbf{q} \times \mathbf{r}_{12}\right]^{4}\right\}^{d}}\right.$,
$d=N_{f}^{2} T k_{0} / \pi \lambda_{1}, \quad k_{0} \sim k_{F}$.

So at finite temperatures the quarkyonic crystal melts into an Abelian critical phase with wave vectors $2 N_{f}$ times greater than the ones established by the energetics at zero temperature. It is essentially a density wave of a quasicondensate of $4 N_{f}$ bound states of quarks. These bound states are spin and flavor singlets.

\section{MAGNETIC FIELD}

A sufficiently strong magnetic field [137] has a profound effect on the structure of the quarkyonic phase. For other, related analyses, see Refs. [138-143].

We start our analysis at zero temperature when the $S U\left(2 N_{f}\right)$ symmetry is spontaneously broken. In this case the matrix $G_{Q}$ can be approximated as

$$
G_{Q}(r)=G_{0} \mathrm{e}^{i t_{Q}^{F}(r) \hat{T}^{F}} \approx G_{0}\left(1+i t_{Q}^{F}(r) \hat{T}^{F}\right),
$$


where $G_{0}$ is some constant matrix and $\hat{T}^{F}$ are generators of the $S U(4)$ algebra. Then to leading order, instead of Eq. (19) to quadratic order we obtain

$$
\begin{aligned}
\mathcal{L}= & V_{0} t^{F} \operatorname{Tr}\left[\hat{T}^{F} \hat{q}(\boldsymbol{\sigma} \mathbf{B}) \hat{T}^{F^{\prime}} \hat{q}(\boldsymbol{\sigma} \mathbf{B})\right] t^{F^{\prime}}+\partial_{\tau} t^{F} \partial_{\tau} t^{F} \\
& +\lambda_{1} t^{F} \operatorname{Tr}\left\{\hat{T}^{F}\left[\lambda_{1}(\mathbf{q} \cdot \mathbf{D})^{2}+\lambda_{2}[\mathbf{q} \times \mathbf{D}]^{4}\right] \hat{T}^{F^{\prime}}\right\} t^{F^{\prime}} .
\end{aligned}
$$

$V_{0} \sim \Delta^{2} / \mu$ is proportional to the square of amplitude of $A_{Q}$ in Eq. (16).

For $N_{f}=2$ it is convenient to represent the generators $T^{a}$ in terms of the Pauli matrices acting in the spin and the flavor spaces:

$$
T^{s}=\left(\sigma^{a} \otimes I\right), \quad T^{f}=\left(I \otimes \tau^{a}\right), \quad T^{(s, f)}=\left(\sigma^{a} \otimes \tau^{b}\right) .
$$

The magnetic field splits the dispersion of the Goldstone modes. In particular, the mode $\left(I \otimes \tau^{z}\right)$ is not affected by the magnetic field and remains gapless. The three $\left(\sigma^{a} \otimes I\right)$ modes and three $\left(\tau^{z} \otimes \sigma^{a}\right)$ are affected only by the Zeeman term. Their spectrum is

$$
E^{2}=\lambda_{1}\left(p_{\|}^{2}+\frac{p_{\perp}^{4}}{k_{0}^{2}}\right)+V_{0} \sum_{f} q_{f}^{2} \mathbf{B}^{2} .
$$

The only modes affected by the orbital magnetic field are the eight modes with off-diagonal $\tau^{ \pm}$Pauli matrices. To simplify the discussion of their spectrum we will consider two limiting cases.

1. $\mathbf{B}\|\mathbf{Q}\| \hat{z}, A_{x}=B y, A_{y}=A_{z}=0$. Then the spectrum is determined by the equation

$$
E^{2} \mathbf{t}=\lambda_{1}\left\{p_{z}^{2}+\frac{1}{k_{0}^{2}}\left[\partial_{y}^{4}+\left( \pm p_{x}-B y\right)^{4}\right]\right\} \mathbf{t} .
$$

Here the real vector $\mathbf{t}$ includes the modes corresponding to generators $\left(I \otimes \tau^{ \pm}\right),\left(\sigma^{a} \otimes \tau^{ \pm}\right)$. We need just to shift $y$ by $\pm p_{x} / B$ after which $p_{x}$ drops out of the eigenvalue equation. The general solution of Eq. (28) is given by

$$
\begin{aligned}
\mathbf{t} & =\Re e\left[\mathbf{f}\left(y B^{1 / 2} \pm p_{x} / B^{1 / 2}\right) \mathrm{e}^{\mathrm{i}\left(p_{x} x+p_{z} z\right)}\right], \\
E^{2} & =\lambda_{1}\left(p_{z}^{2}+\frac{B^{2} \epsilon^{2}}{k_{0}^{2}}\right), \\
\epsilon^{2} \mathbf{f}(\tau) & =\left(\partial_{\tau}^{4}+\tau^{4}\right) \mathbf{f}(\tau) .
\end{aligned}
$$

The spectrum is

$$
E^{2}=\lambda_{1}\left[p_{z}^{2}+\frac{B^{2} g_{1}(n)}{k_{0}^{2}}\right]
$$

We can determine function $g_{1}(n)$ at large quantization numbers $n \gg 1$ using semiclassical approximation:

$$
\mathbf{f} \sim \mathbf{f}_{0} \exp \left[\mathrm{i} \int_{-\sqrt{\epsilon}}^{\tau} d t\left(\epsilon^{2}-t^{4}\right)^{1 / 4}\right]
$$

Then the spectrum follows from the Bohr-Sommerfeld quantization condition:

$$
\int_{-\sqrt{\epsilon}}^{\sqrt{\epsilon}} d t\left(\epsilon^{2}-t^{4}\right)^{1 / 4}=\pi n
$$

so that at $n \gg 1$

$$
g_{1}(n)=(\gamma n)^{2}, \quad \gamma=\pi / \int_{-1}^{1}\left(1-x^{4}\right)^{1 / 2} d x .
$$

2. $\mathbf{B} \perp \mathbf{Q}$ and $\| \hat{y}, A_{x}=B z, A_{y}=A_{x}=0$.

$$
E^{2} \mathbf{t}=\lambda_{1}\left\{-\partial_{z}^{2}+k_{0}^{-2}\left[\left(p_{x} \pm B z\right)^{4}+p_{y}^{4}\right]\right\} \mathbf{t} .
$$

The spectrum is given by

$$
E^{2}=\lambda_{1} k_{0}^{-2}\left[p_{y}^{4}+\left(k_{0} B\right)^{4 / 3} g_{2}(n)\right],
$$

where using the semiclassical approximation at $n \gg 1$ we get

$$
g_{2}(n)=(\eta n)^{4 / 3}, \quad \eta=\pi / \int_{-1}^{1}\left(1-x^{4}\right)^{1 / 2} d x .
$$

To summarize: in the presence of a magnetic field, the spectrum is divided into three groups. There are eight gapped modes which include components along the $\tau^{ \pm}$ generators; their gaps depend strongly on the direction of the magnetic field. The second group consists of the modes which include only spin operators and $\tau^{z}$; they have much smaller gaps $\sim B(\Delta / \mu)$. The third group includes the mode $\left(I \otimes \tau^{z}\right)$ which remains gapless.

Hence there are three regimes of temperature. When temperature is so high that the correlation length is smaller than the magnetic length the influence of the magnetic field is small:

$$
\exp \left(-C \Lambda^{2} / N_{f} m T\right)>B \mu_{B} / k_{0} v_{F} .
$$

There is an intermediate interval of temperature when the magnetic field suppresses some modes, leaving as nominally gapless the modes $\left(I \otimes \tau^{z}\right),\left(\sigma^{a} \otimes I\right)$ and $\left(\sigma^{a} \otimes \tau^{z}\right)$. In this region one can approximate the order parameter field as a product of $\mathrm{SU}(2)$ matrix $g$ and $\mathrm{U}(1)$ matrix $\mathrm{V}: G=$ $g_{\mathrm{SU}(2)} V$ with $V=\cos \alpha I+\mathrm{i} \tau^{z} \sin \alpha$. The $\mathrm{SU}(2)$-symmetric part of the order parameter is disordered by thermal fluctuations, as was demonstrated in Sec. IV. The action for the $\mathrm{U}(1)$ part is Gaussian and the field $\alpha$ has long range correlations. This is in addition to the overall $\mathrm{U}(1)$ phase $\phi$.

At yet smaller temperatures the $\mathrm{SU}(2)$ part $g$ is gapped by the magnetic field, through quantum effects. In both 
cases the Abelian modes $\alpha, \phi$ (the total charge and the diagonal flavor one) survive as gapless. Being Abelian they remain long ranged even if fluctuations are taken into account. As a consequence by gapping out all non-Abelian modes the magnetic field instigates quasi long range order in the $\sum_{\sigma} \rho_{(f, \sigma),(f, \sigma)}$ which is quadratic in quark creation and annihilation operators.

\section{PHASE DIAGRAMS WITH A LIFSHITZ POINT}

\section{A. General effective Lagrangian}

In the previous section we considered chiral spirals in a quarkyonic phase in QCD. This is relevant at chemical potentials above that for hadronic nuclear matter, but below those where perturbative QCD applies.

Moving up in chemical potential, the transition from a quarkyonic phase to the perturbative regime appears straightforward. The width of a patch with a chiral spiral is proportional to the square root of the string tension, Eq. (9). As discussed in Sec. II, numerical simulations for two colors show that the string tension decreases with increasing chemical potential, and is essentially zero by $\mu_{q} \sim 750 \mathrm{MeV}$, Fig. 3 of Ref. [123]. In a perturbative regime the interactions near the Fermi surface lead to color superconductivity symmetrically over the Fermi surface, instead of leading to formation of chiral spirals in patches. On the quarkyonic side the only order parameter is the $U(1)$ phase of the determinantal operator, $\operatorname{det} \hat{\rho}_{\mathbf{Q}}$ of Eq. (22). On the color superconductivity side the broken symmetry is completely different and therefore it is natural to expect that the phase transition is of first order, as between charge density wave and superconductivity in condensed matter systems. There are also other order parameters for color superconductivity which may enter [144]. For three colors the precise value at which this transition occurs for three colors cannot be fixed by our qualitative arguments, but following the discussion at the beginning of Sec. II, it is presumably somewhere around $\mu_{q} \sim 1 \mathrm{GeV}$.

Going down in density, from the quarkyonic phase to hadronic nuclear matter, there are chiral spirals in the former, and pion/kaon condensates in the latter. In terms of the usual chiral order parameter,

$$
\rho=\bar{q}_{L} q_{R}
$$

the condensate between $\sigma$ and $\pi^{3}$ is given by

$$
\rho_{\pi \mathrm{cond}}=\rho_{0} \exp \left(i(\mathbf{Q} \cdot \mathbf{r}+\phi) t_{3}\right),
$$

where $\rho_{0}$ is a constant $\sim f_{\pi}$, and $t_{3}$ is a flavor matrix. In neutron stars, with a charged background of protons the analogous condensates are along the directions corresponding to $\pi^{-}$and $K^{-}$[26-43].
This suggests that there is a direct relation between the pion/kaon condensates of hadronic nuclear matter and those of the quarkyonic regime. We argued previously that the only true order parameter for the quarkyonic regime was an overall phase factor of $U(1)$. Such a phase clearly arises for the field of Eq. (39), which we denote by the phase $\phi$. The physical origin of this phase is obvious: at a given point along the $\mathbf{Q}$ direction, the condensate points entirely in a given direction, say along $\pi^{3}$. Where this point is just an overall shift in the phase, though.

Thus pion/kaon condensates have a $U(1)$ phase, which is sometimes termed the "phonon" mode [96,98]. This is then a strict order parameter which distinguished hadronic nuclear matter, without a pion/kaon condensate, from that with. If this transition is not of first order, then it must be of second order, in the universality class of $U(1)$.

As the chemical potential increases further, it is very plausible that it is not possible to rigorously distinguish between the pion/kaon condensate of Eq. (39) and chiral spirals of the quarkyonic regime. The only difference is that there are $N_{f}^{2}-1$ very light modes for a pion/kaon condensates, and $4 N_{f}^{2}-1$ light modes for a quarkyonic chiral spiral. It is natural to assume that the additional $3 N_{f}^{2}$ modes of the latter become lighter as $\mu_{q}$ increases.

As discussed previously, there may be phase transitions as the number of patches increases, although this is not really necessary. In that vein, we note that in the original discussion of pion condensates by Overhauser [26], it was explicitly stated that the simplest solution in three dimensions is that with six patches.

The relation between pion/kaon condensates and chiral spirals also suggests a less trivial speculation. For static quantities, at high density the effective theory for the light modes of a chiral spiral is a $S U\left(2 N_{f}\right)$ sigma model. Once transverse fluctuations (for massless quarks) are included, or magnetic interactions (for massive), as we argued in Sec. III, $S U\left(2 N_{f}\right)$ sigma model reduces to a $S U\left(N_{f}\right)$ model. This agrees with the effective theory for a pion/kaon condensate, which is a nonlinear sigma model on $S U\left(N_{f}\right)$.

However, we argued that for nonstatic quantities, there is also a WZNW term, of level 3 for $S U\left(2 N_{f}\right)$, and level 6 for $S U\left(N_{f}\right)$. This suggests that there might be a WZNW term for pion/kaon condensates, with level 6. This is not obvious. The original theory has a WZNW term in four dimensions, but this involves derivatives in all four dimensions [145]. Perhaps a WZNW term arises in two dimensions from the variation of the patches in the transverse directions.

The similarity between chiral spirals and pion/kaon condensates has been recognized previously, at least implicitly [93-95,97]. The appearance of a $U(1)$ order parameter, and the possible appearance of a WZNW term, is novel. 
To support the above considerations we establish a formal correspondence between the relativistic and the nonrelativistic versions of the QCD Hamiltonian. We start with the Dirac Hamiltonian:
$H=\hat{q}^{+}(\mathbf{p})\left(\hat{\tau}_{z} \otimes \hat{\sigma}^{a} p^{a}+m \hat{\tau}^{x} \otimes \hat{I}-\mu \hat{I} \otimes \hat{I}\right) \hat{q}(\mathbf{p})$,

where $\tau^{a}$ act in the chiral basis $(R, L)$ and $\sigma^{a}$ act in the spin space. We neglect the quark mass. Then under the transformation

$$
\begin{aligned}
\left(\begin{array}{c}
q_{R \sigma}(\mathbf{p}) \\
q_{L \sigma}(\mathbf{p})
\end{array}\right)= & \frac{1}{\sqrt{2}}\left(\begin{array}{c}
z_{\sigma}(\mathbf{n}) \\
\epsilon_{\sigma \sigma^{\prime}} z_{\sigma^{\prime}}^{*}(\mathbf{n})
\end{array}\right) \psi_{+}(p)+\frac{1}{\sqrt{2}}\left(\begin{array}{c}
\epsilon_{\sigma \sigma^{\prime}} z_{\sigma^{\prime}}^{*}(\mathbf{n}) \\
z_{\sigma}(\mathbf{n})
\end{array}\right) \psi_{-}(\mathbf{p}) \\
& +\frac{1}{\sqrt{2}}\left(\begin{array}{c}
z_{\sigma}(\mathbf{n}) \\
-\epsilon_{\sigma \sigma^{\prime}} z_{\sigma^{\prime}}^{*}(\mathbf{n})
\end{array}\right) \eta_{-}^{+}(p)+\frac{1}{\sqrt{2}}\left(\begin{array}{c}
\epsilon_{\sigma \sigma^{\prime}} z_{\sigma^{\prime}}^{*}(\mathbf{n}) \\
-z_{\sigma}(\mathbf{n})
\end{array}\right) \eta_{+}^{+}(\mathbf{p}),
\end{aligned}
$$

where

$z^{+} \boldsymbol{\sigma} z=\mathbf{n} \equiv \frac{\mathbf{p}}{p}, \quad z_{\sigma}(\mathbf{n})=\epsilon_{\sigma \sigma^{\prime}} z_{\sigma^{\prime}}^{*}(-\mathbf{n}), \quad \sum_{\sigma= \pm 1} z_{\sigma}^{*} z_{\sigma}=1$,

the Hamiltonian Eq. (40) becomes

$H=\sum_{\mathbf{p}, \tau= \pm}\left[(|p|-\mu) \psi_{\tau}^{+}(\mathbf{p}) \psi_{\tau}(\mathbf{p})+(|p|+\mu) \eta_{\tau}^{+}(\mathbf{p}) \eta_{\tau}(\mathbf{p})\right]$.

In what follows we will drop the antiparticles $\eta$.

Now let us consider the chiral order parameter $\rho$ selecting in it only the part associated with particles:

$$
\begin{aligned}
\rho & =q^{+}(r) \tau^{x} q \\
& =\frac{1}{2} \sum_{p, p^{\prime}} \mathrm{e}^{\mathrm{i}\left(\mathbf{p}-\mathbf{p}^{\prime}\right) \mathbf{r}} \psi_{\alpha}^{+}(p) \psi_{\beta}\left(p^{\prime}\right)\left(\mathbf{e}_{\alpha}^{*}(p) \mathbf{e}_{\beta}\left(p^{\prime}\right)\right)+\cdots \\
& =\frac{1}{2} \sum_{Q, p} \mathrm{e}^{\mathrm{i} \mathbf{Q} \mathbf{r}_{\alpha}} \psi_{\alpha}^{+}(\mathbf{Q} / 2+\mathbf{p}) \psi_{\alpha}(-\mathbf{Q} / 2+p)+\cdots,
\end{aligned}
$$

where the basis vectors e are defined in (41) and the ellipses include the contributions of antiparticles. This shows that the chiral order parameter $\rho$, which is uniform at $\mu=0$, naturally acquires oscillatory terms at $\mu \neq 0$. These can be either pion/kaon condensates or quarkyonic chiral spirals. As we neglected antiparticles, this only happens for sufficiently large $\mu_{q}$. These arguments do not show how large $\mu_{q}$ must be, but they do establish that both chiral symmetry breaking and the formation of inhomogeneous phases can be described within the same effective model.

To discuss the phase diagram we then consider a $S U\left(N_{f}\right) \times S U\left(N_{f}\right)$ field $\rho$, taking a customary linear sigma model,

$$
\begin{aligned}
\mathcal{L}= & \frac{1}{2} \operatorname{tr}\left|\partial_{0} \rho\right|^{2}+\frac{\mathcal{Z}}{2} \operatorname{tr}\left|\partial_{i} \rho\right|^{2}+\frac{1}{2 \mathcal{M}^{2}} \operatorname{tr}\left|\partial^{2} \rho\right|^{2} \\
& +\frac{m^{2}}{2} \operatorname{tr} \rho^{\dagger} \rho+\frac{\lambda_{1}}{4}\left(\operatorname{tr} \rho^{\dagger} \rho\right)^{2}+\frac{\lambda_{2}}{4} \operatorname{tr}\left(\rho^{\dagger} \rho\right)^{2}+\frac{\kappa}{6}\left(\operatorname{tr} \rho^{\dagger} \rho\right)^{3}+\ldots
\end{aligned}
$$

In four spacetime dimensions $\rho$ has dimensions of mass. The first two terms are standard kinetic terms. The coefficient of the second term, with two spatial derivatives, can have an arbitrary coefficient $\mathcal{Z}$. Implicitly we consider systems at nonzero temperature and quark density, and so there is a preferred rest frame. Then Lorentz symmetry is lost, and $\mathcal{Z} \neq 1$ is allowed. In particular, we allow $\mathcal{Z}$ to be negative. Stability then requires the addition of a positive term with four spatial derivatives; the coefficient of that term is $\sim 1 / \mathcal{M}^{2}$, where $\mathcal{M}$ is some mass scale derived from the underlying theory.

While we only consider static quantities, and so can ignore the first term with two time derivatives, we add it to emphasize that the only higher derivatives considered are those in the spatial coordinates. It is well known that higher order derivatives in time lead to acausal behavior, which should not occur in an effective theory. This is not dissimilar to causal theories of higher derivative gravity, such as Horava-Lifshitz gravity [146-149].

When the coefficient of the term with two spatial derivatives is positive, $\mathcal{Z}>0$, the phase diagram is standard. For positive mass squared, $m^{2}>0$, the theory is in a symmetric phase, with $\langle\rho\rangle=0$. For positive quartic coupling, $\lambda>0$, the global flavor symmetry is broken when the mass squared is negative, $m^{2}<0$, but not the translational symmetry. At zero mass, $m^{2}=0$, there is a second order transition in the appropriate universality class.

The hexatic couplings, such as $\kappa$, are assumed to be positive, so the quartic coupling $\lambda$ can be negative. It is easy to show that there is then a first order transition at positive mass squared, $m^{2}>0$.

In the plane of the mass squared, $\mathrm{m}^{2}$, and the quartic couplings $\lambda$, the phase diagram is standard. For positive $\lambda$ there is a line of second order transitions when $m^{2}=0$. For 
negative $\lambda$ there is a line of first order transition at positive $m^{2}$. These two lines meet at the origin, $m^{2}=\lambda=0$, which is a tricritical point. In three dimensions the critical exponents for a tricritical point are those of mean field, with logarithmic corrections controlled by the hexatic interactions [150].

Since there is more than one quartic coupling, even if the quartic couplings are originally positive, in the infrared limit they can flow to negative values, and so generate a fluctuation induced first order transition [150-153]. For this Lagrangian in $4-\epsilon$ dimensions, to leading order in $\epsilon$ this happens when $N_{f}>\sqrt{2}$ [154]. This is only valid to leading order in $\epsilon$. For two flavors, the symmetry is $S U(2)_{L} \times S U(2)_{R} \times U(1)_{A} \equiv O(4) \times O(2)$, then the conformal bootstrap program suggests that there is a nontrivial fixed point in three dimensions, $\epsilon=1$, which is not present for small $\epsilon[155,156]$. If true, then in the plane of $m^{2}$ and $\lambda$, there are two lines of first order transitions which meet at a tricritical point.

We next consider the corresponding phase diagram in the plane of $m^{2}$ and the wave function renormalization constant $\mathcal{Z}$. The case of positive $\mathcal{Z}$ is a trivial consequence of the above analysis, with a line of second order transitions along $m^{2}=0$.

We next turn to the case of $m^{2}<0$, when $\rho$ has an expectation value $\rho_{0} \neq 0$. Spatially inhomogeneous condensates arise when $\mathcal{Z}$ is negative. We take

$$
\rho_{C S}=\rho_{0} \exp (i \mathbf{Q} \cdot \mathbf{x})
$$

where $\mathbf{Q}=Q_{0} \hat{z}$.

The ansatz of Eq. (46) is only a caricature of the full solution. The detailed form of the condensate differs depending upon whether the broken symmetry is discrete or continuous. For a discrete $Z(2)$ symmetry, the condensate is a kink crystal, where the field oscillates in sign in one direction, as in Eq. (46). For a continuous symmetry of $U(1)$, the condensate is a spiral, where the field oscillates in two directions, as in Eq. (39). These differences are illustrated by the exact solutions in $1+1$ dimensions: for an infinite number of flavors, at low $T$ and nonzero $\mu$ the Gross-Neveu model develops a kink crystal, while the chiral Gross-Neveu model has a chiral spiral [44-49]. The condensates for more complicated continuous symmetries can be more involved, as for Eq. (6).

For our qualitative analysis, though, the precise form of the condensate is secondary. We then minimize the terms with spatial derivatives with respect to $Q_{0}$,

$$
Q_{0}^{2}=-2 \mathcal{Z} \mathcal{M}^{2}
$$

For $Q_{0}$ to be real, $\mathcal{Z}$ has to be negative. Plugging this back into the Lagrangian, we obtain

$$
\mathcal{V}=\frac{1}{2} m_{\mathrm{eff}}^{2} \rho^{2}+\frac{\lambda}{4}\left(\rho^{2}\right)^{2},
$$

where $\lambda=N_{f}^{2} \lambda_{1}+N_{f} \lambda_{2}$, and

$$
m_{\text {eff }}^{2}=m^{2}-\frac{\mathcal{Z}^{2}}{4} \mathcal{M}^{2}
$$

Henceforth we ignore the hexatic coupling $\sim \kappa$, as we uniformly assume that the quartic coupling $\lambda$ is positive.

Consider a given value of $m^{2}<0$, crossing from positive to negative values of $\mathcal{Z}$. For $\mathcal{Z}>0$, the theory is in a broken phase; for negative $\mathcal{Z}$, in the phase with the chiral spiral of Eq. (46). Ignoring $\kappa$, the potential energy at the minimum is

$$
\mathcal{V}=-\frac{1}{4 \lambda} m_{\mathrm{eff}}^{4}
$$

Assuming that the variation in $\mathcal{Z}$ is linear in the appropriate thermodynamic variable, such as the chemical potential or temperature $T$,

$$
\mathcal{Z}=z_{0}\left(T-T_{0}\right)
$$

then it is trivial to show that while the potential, or free energy, is continuous at $T=T_{0}$, the first derivative, related to the energy density, is discontinuous. This is natural: except for Goldstone bosons, the correlation lengths are nonzero in both phases. Further, there is an order parameter which distinguishes the phases: $\langle\phi\rangle$ is constant when $\mathcal{Z}$ is positive, while with the chiral spiral of Eq. (46), the spatial average of $\langle\phi\rangle$ vanishes when $\mathcal{Z}<0$.

Consider next the case when $\mathcal{Z}$ is negative and the original mass squared, $m^{2}$, is positive. Then the effective mass $m_{\text {eff }}$ vanishes when $m^{2}=\mathcal{Z}^{2} \mathcal{M}^{2} / 4$, and one expects a second order phase transition.

It was shown by Brazovskii [89,107-109] that instead there is a first order transition. For the assumed parameters, the $\phi$ propagator is

$$
\Delta^{-1}(\boldsymbol{k})=m^{2}+\mathcal{Z} \boldsymbol{k}^{2}+\frac{\left(\boldsymbol{k}^{2}\right)^{2}}{\mathcal{M}^{2}} .
$$

When $\mathcal{Z}<0$, there is a minimum for nonzero spatial momentum. Expand

$$
\boldsymbol{k}=\left(k_{\mathrm{tr}}, Q_{0}+k_{z}\right) .
$$

Inserting Eq. (53) into Eq. (52) and expanding, the terms proportional to $k_{z}$ vanish if $Q_{0}$ satisfies Eq. (47). Then

$$
\Delta^{-1}(\boldsymbol{k}) \approx m_{\mathrm{eff}}^{2}-2 \mathcal{Z} k_{z}^{2}+\ldots,
$$

where $m_{\text {eff }}^{2}$ is that of Eq. (49). Notice that the terms quadratic in the transverse momenta, $k_{\text {tr }}$, vanish, although 
there are terms of higher order in $k_{\text {tr }}$. This is similar to the behavior of Goldstone bosons in a chiral spiral.

Consequently, an integral over virtual fields is dominated by fluctuations in the direction of $k_{z}$. When the effective mass is small, the correction to the mass term is

$\Delta m^{2} \sim \lambda T \int \frac{d^{3} k}{(2 \pi)^{3}} \frac{1}{m_{\mathrm{eff}}^{2}+\left(k^{2}-Q_{0}^{2}\right)^{2} / \mathcal{M}^{2}} \sim+\frac{T \lambda Q_{0} \mathcal{M}}{m_{\mathrm{eff}}}$.

Similarly, the correction to the quartic coupling is

$\Delta \lambda \sim-\lambda^{2} T \int d^{3} k \frac{1}{\left(m_{\text {eff }}^{2}+\left(k^{2}-Q_{0}^{2}\right)^{2} / \mathcal{M}^{2}\right)^{2}} \sim-\frac{\lambda^{2} T Q_{0} \mathcal{M}}{m_{\text {eff }}^{3}}$.

Both of these results follow because the fluctuations for small $m_{\text {eff }}$ are those for a theory in one dimension, along $k_{z}$. Because the infrared divergences of Eqs. (55) and (56) bring in powers of $1 / m_{\text {eff }}$, a second order transition, where $m_{\text {eff }}=0$, is not possible. There is a transition between the two phases, but it is necessarily of first order, where $m_{\mathrm{eff}}$ is always nonzero in each phase.

This has been termed a "fluctuation induced first order" transition [89,107-109], but the terminology is somewhat misleading. In theories with several coupling constants, couplings can flow to negative values [150-153], and so generate a first order transition. This depends upon how the coupling constants flow under the renormalization group in the infrared limit, and so depends both upon the symmetry group, and the dimensionality of space-time.

In contrast, what happens for $m^{2}>0$ and $\mathcal{Z}<0$ is just an effective reduction of the fluctuations to one dimension. It does not depend upon either the global symmetry or the original dimensionality of spacetime.

This yields the mean field diagram of Fig. 1. In the plane of $m^{2}$ and $\mathcal{Z}$, the broken phase with $\langle\phi\rangle \neq 0$ in the upper left hand quadrant; the symmetric phase, $\langle\phi\rangle=0$, in the upper right hand quadrant and part of the lower right hand quadrant, and the remainder the phase with a chiral spiral. They meet at the origin, $m^{2}=\mathcal{Z}=0$, which is the Lifshitz point.

Consider the theory at the Lifshitz point. The static propagator is

$$
\Delta(\boldsymbol{k})=\frac{\mathcal{M}^{2}}{\left(\boldsymbol{k}^{2}\right)^{2}} .
$$

At leading order the leading correction to the mass is

$$
\Delta m^{2} \sim-\lambda \int d^{d} k \frac{\mathcal{M}^{2}}{\left(\boldsymbol{k}^{2}\right)^{2}+m^{2} \mathcal{M}^{2}} .
$$

This develops a logarithmic divergence in the infrared in four dimensions, which is then the lower critical dimension

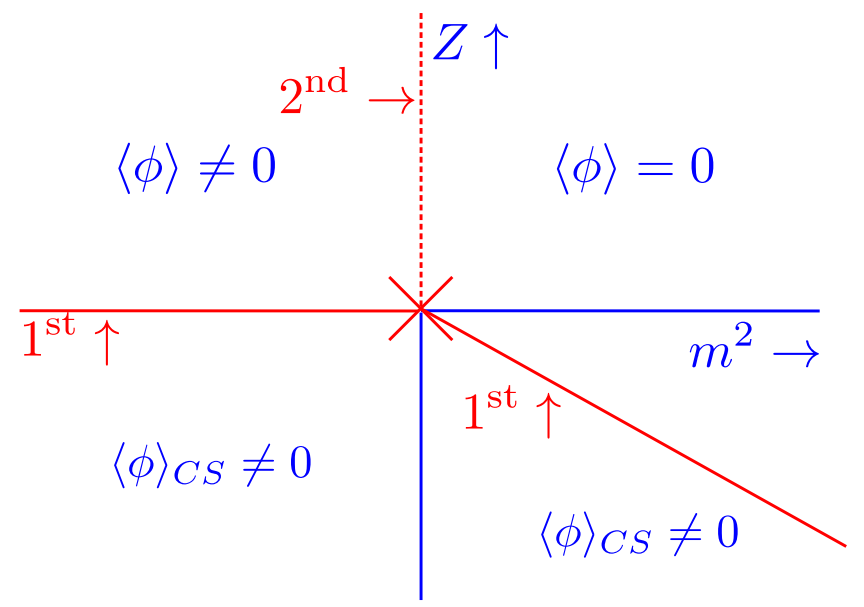

FIG. 1. The phase diagram in mean field theory, in the plane of $\mathrm{m}^{2}$ and $\mathcal{Z}$ : a line of second order transitions, meeting two lines of first order transitions, which meet at the Lifshitz point, where $m^{2}=\mathcal{Z}=0$.

[15-17,23,24]. Corrections to the quartic coupling begin at one loop order as

$$
\Delta \lambda \sim-\lambda^{2} \int d^{d} k \frac{\mathcal{M}^{4}}{\left(\boldsymbol{k}^{2}\right)^{2}\left((\boldsymbol{k}-\boldsymbol{p})^{2}\right)^{2}} .
$$

This is logarithmically divergent in eight dimensions, which is the upper critical dimension [15-17]. This is in contrast to an ordinary critical point: for a propagator $\Delta(k)=1 / k^{2}$, where the lower and upper critical dimensions are two and four, respectively.

At the Lifshitz point in four spatial dimensions, in the infrared the logarithmic divergences always disorder the theory. This is stronger at nonzero temperature, when $d=3$ and the infrared divergences are power like $\sim 1 / \mathrm{m}$. Consequently, once fluctuations are included, there cannot be a true Lifshitz point.

Inhomogeneous polymers provide an example of the absence of a Lifshitz point in three spatial dimensions [18-22]. The simplest case is a mixture of oil and water. These separate into droplets of oil or water, but by adding a surfacant to alter the interface tension, other phases emerge. A related example is a mixture of two different polymers, formed from monomers of type $\mathrm{A}$ and type $\mathrm{B}$. To this are added A-B diblock copolymers, which are long sequences of type A, followed by type B. These A-B copolymers localize at the interfacial boundaries separating phases with only A or B homopolymers, and act to decrease the interface tension; at sufficiently high concentrations, the interface tension changes sign, and is negative.

By varying the temperature and the concentration of diblock copolymers one can form three different phases. At high temperature $\mathrm{A}, \mathrm{B}$, and $\mathrm{A}-\mathrm{B}$ polymers mingle to form a homogeneous phase, analogous to the symmetric phase of a spin system. At low temperature and low concentrations of 
A-B copolymers, the system separates into droplets of A, B and A-B polymers, which is like the broken phase of a spin system. At low temperature and high concentration of A-B copolymers, the interface tension becomes negative, and there is an inhomogeneous phase, as the system forms a lamellar state with alternating layers of A and B polymers. This is similar to a smectic liquid crystal, albeit without orientational order.

Mean field theory predicts that there is a Lifshitz point where these three phases meet. In contrast, both experiment and numerical simulations with self-consistent field theory indicate that there is no Lifshitz point [18-22]: see, e.g., Fig. 3 of Ref. [21]. Instead, the symmetric phase enlarges, and includes a bicontinuous microemulsion, which exhibits nearly isotropic fluctuations in composition with large amplitude. In this regime the surface tension is essentially zero, and there is a spongelike structure with large entropy.

The absence of the Lifshitz point can be understood by analogy. Consider a spin system, with a continuous symmetry, in two or fewer dimensions. The symmetry cannot be spontaneously broken as that would generate massless Goldstone bosons, which are not possible in such a low dimensionality. Instead, fluctuations generate a mass nonperturbatively.

What happens in the Lifshitz regime, when the number of spatial dimensions is four or less, is similar. We can tune either the coefficient of the term quadratic in momenta to vanish, $\mathcal{Z}=0$, or the mass, $m^{2}$, to vanish, but not both. If $m^{2}=0$, then $\mathcal{Z} \neq 0$ is generated nonperturbatively; alternately, if $\mathcal{Z}=0$, then $m^{2} \neq 0$ is generated nonperturbatively. For the latter, the propagator is not Eq. (57), but

$$
\Delta(\boldsymbol{k})=\frac{\mathcal{M}^{2}}{\left(\boldsymbol{k}^{2}\right)^{2}+m^{2} \mathcal{M}^{2}},
$$

where $m^{2} \neq 0$ is nonperturbative. We cannot conclude anything about the size of the Lifshitz regime, only that it exists. For inhomogeneous polymers, the Lifshitz regime includes a bicontinuous microemulsion, where $\mathcal{Z} \approx 0$ and $m^{2} \neq 0$; see, e.g., Fig. 2 of Ref. [21].

A possible phase diagram which incorporates fluctuations is that of Fig. 2. There is a strict order parameter which distinguishes the broken and symmetric homogeneous phases, so the line of second order transitions must intersect the line of first order transitions. They do so at a Lifshitz critical endpoint $\tilde{\mathcal{C}}$. By continuity, as $\tilde{\mathcal{C}}$ is approached along the line of first order transitions, the latent heat vanishes.

Consider the usual phase diagram where a line of second order transitions meets a line of first order transitions at a critical endpoint $\mathcal{C}$. The universality class along the line of second order transitions is determined by the unbroken symmetry group and the dimensionality of space, with nonzero values for the quartic couplings of Eq. (45), $\lambda \neq 0$. At the critical endpoint $\mathcal{C}$, the quartic couplings vanish,

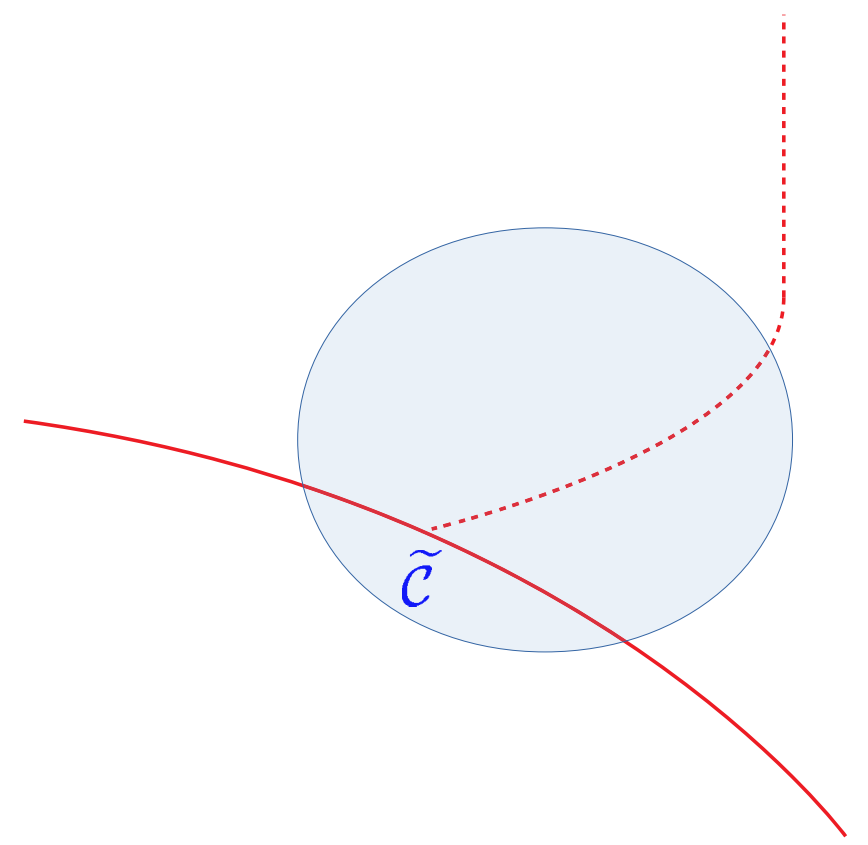

FIG. 2. The Lifshitz phase diagram corrected by fluctuations: the line of second order transitions still intersects the line of first order transitions, but one cannot reach the Lifshitz point, where $\mathcal{Z}=m^{2}=0$. The shaded region denotes the Lifshitz regime, where there are large infrared fluctuations. The line of second order transitions meets the line of first order transitions at the Lifshitz critical endpoint $\tilde{\mathcal{C}}$.

$\lambda=0$, and the hexatic couplings $\kappa$ dominate. This changes the upper critical dimensionality from four to three.

The Lifshitz critical endpoint $\tilde{\mathcal{C}}$ is not of this form. The simplest possibility is that at $\tilde{\mathcal{C}}$, a term quadratic in the momenta, $\mathcal{Z}>0$, is generated non perturbatively, with $m^{2}=0$. This implies that the universality class of the Lifshitz critical endpoint $\tilde{\mathcal{C}}$ is the same as along the line of second order transitions.

Consider moving away from the Lifshitz critical endpoint $\tilde{\mathcal{C}}$, down in $\mathcal{Z}$ into the inhomogeneous phase. Since mean field theory indicates that an inhomogeneous phase only arises when $\mathcal{Z}$ is negative, the appearance of an inhomogeneous phase infinitesimally below $\tilde{\mathcal{C}}$ must be due to strong, nonperturbative fluctuations.

Alternately, consider moving away from the Lifshitz critical endpoint to the right, for increasing $\mathrm{m}^{2}$. Doing so, one will enter a region where $\mathcal{Z}$ is very small, but the mass squared $m^{2}$ is nonzero and positive. This region is directly analogous to a bicontinuous microemulsion [18-22]. For inhomogeneous polymers, this region is seen to be an enlargement of the symmetric phase into the region between the inhomogeneous and broken phases. This explains the curvature of the line of second order transitions in Fig. 2. We do not explicitly indicate the axes $\mathcal{Z}$ and $m^{2}$ in Fig. 2 because the Lifshitz point of mean field theory, $\mathcal{Z}=m^{2}=0$, is not accessible physically. 
We note that the phase diagram of mean field theory is correct in a limit without fluctuations. Examples include Gross-Neveu type models in two spacetime dimensions, which are soluble for an infinite number of flavors, $N=\infty$ [44-49]. At large but finite $N$, then, the width of the Lifshitz regime is automatically $\sim 1 / N$. It would be useful to study the Lifshitz regime in models with a large $N$ expansion, both in the lower critical dimension of four and below four dimensions. This would provide a test of the Lifshitz phase diagram in Fig. 2 and especially of the universality class of the Lifshitz critical endpoint $\tilde{\mathcal{C}}$.

Before continuing to the implications for the phase diagram of QCD, we remark that our analysis is valid for nonzero temperature in three spatial dimensions. At zero temperature, by causality there must always be terms quadratic in the energy. The integral analogous to Eq. (58) then becomes

$$
\begin{aligned}
\Delta m^{2} & \sim-\lambda \int d \omega \int d^{d} k \frac{1}{\omega^{2}+\left(\boldsymbol{k}^{2}\right)^{2} / \mathcal{M}^{2}+m^{2}} \\
& \sim-\lambda \int d^{d} k \frac{\mathcal{M}}{\sqrt{\left(\boldsymbol{k}^{2}\right)^{2}+m^{2} \mathcal{M}^{2}}} .
\end{aligned}
$$

As $m \rightarrow 0$ this is infrared convergent in more than two spatial dimensions, $d>2$. Thus we expect that the infrared fluctuations are well behaved at low temperature. Further, the dynamic behavior near the Lifshitz critical endpoint, $\tilde{\mathcal{C}}$, differs from that for a typical critical endpoint, $\mathcal{C}$.

\section{B. Possible phase diagram of QCD}

The above analysis applies to the chiral limit, where pions are massless in the broken phase and there is a line of second order phase transitions ending in the Lifshitz critical endpoint $\tilde{\mathcal{C}}$. In QCD, with massive pions the line of second order transitions becomes a crossover line.

In contrast, the existence of spatially inhomogeneous phases is not sensitive to moving away from the chiral limit. This was implicit in Sec. II, where we considered massive quarks. It is also seen in solutions of effective models, as demonstrated in effective models [157]. The cleanest example is given by Bringoltz, who solved the 't Hooft model in $1+1$ dimensions for a heavy quark [60] and showed there are oscillations about a nonzero value of $\bar{q} q$.

In general, the phase diagram is a function of at least three parameters: the mass squared, quartic coupling(s), and the spatial wave function renormalization $\mathcal{Z}$. For the purposes of discussion, we assumed previously that the quartic couplings of the effective model remain positive, so there is only a Lifshitz point and not a critical endpoint.

Because of the sign problem, at present this cannot be decided in QCD through numerical simulations. This leaves us with effective models, where the results are suggestive, and not definitive. In the Nambu Jona-Lasino model [95,158] [159], or solutions of Schwinger-Dyson equations [74], the Lifshitz and critical endpoints coincide: see, e.g., Fig. 6 of Buballa and Carignano [95]. This phase diagram is analogous to Fig. 1, where the tricritical point, $m^{2}=\lambda=0$, coincides with the Lifshitz point, $m^{2}=\mathcal{Z}=0$.

This coincidence is not generic, however. Carignano, Buballa, and Schaefer [75] showed that in a quark-meson model, that the critical endpoint and Lifshitz points coincide when the sigma mass is twice the constituent quark mass, $m_{\sigma}=2 m_{\mathrm{qk}}$. In such models it is also possible to allow $m_{\sigma} \neq 2 m_{\mathrm{qk}}$; in that case, the Lifshitz and critical endpoints separate.

There are then two possibilities. Assuming there is a region with spatial inhomogeneities, either the Lifshitz point moves into the region with spatial inhomogeneities, or the critical endpoint does. If the former, fluctuations are dominated by the critical endpoint, which is the standard scenario.

If the former, fluctuations are dominated by the Lifshitz regime, as the would be critical endpoint is in the region with spatial inhomogeneities. The natural analogy of the phase diagram in Fig. 2 is that of Fig. 3.

Numerical simulations search for a critical endpoint from the behavior of the power series in $\mu$ about $\mu=0$ and the associated singularity in the complex $\mu$ plane. For our proposed phase diagram, though, there is only an unbroken line of first order transitions, and no associated singularity [160]. This is in accord with present data from numerical simulations, which do not find evidence for a critical endpoint [161-167]. Of course, it is also possible that the critical endpoint is farther away in the plane of $T$ and $\mu$.

We make one comment about the highest temperature at which there is a spatially inhomogeneous phase, $T_{0}$,

$$
\left.\frac{\partial T}{\partial \mu}\right|_{T_{0}}=0
$$

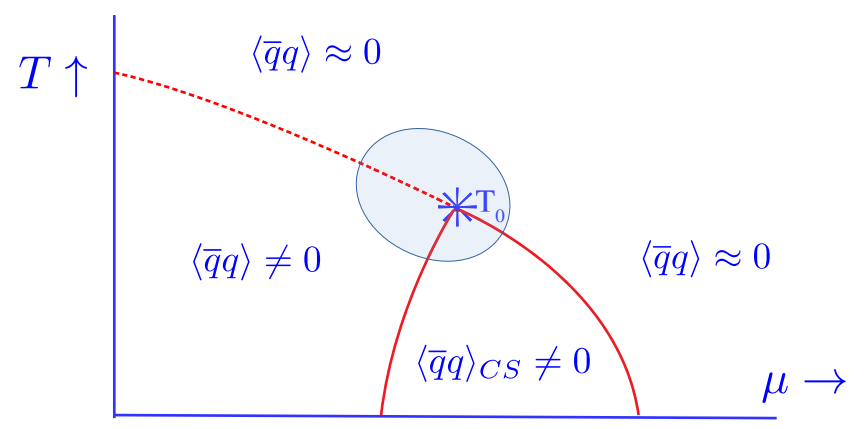

FIG. 3. A proposed phase diagram for QCD: the solid line represents first order transitions which separate homogeneous from spatially inhomogeneous phases; the dashed line, crossover; the shaded region, the Lifshitz regime. The highest temperature at which a spatially inhomogeneous phase occurs defines the point of equal densities, $T_{0}$. We assume that the point where the quartic couplings become negative is in the spatially inhomogeneous phase, where $\langle\bar{q} q\rangle_{C S} \neq 0$, and so does not generate a critical endpoint. 
Since the pressure is continuous at a first order phase transition, by taking derivatives of the pressure with respect to $\mu$, we find

$$
n_{+}=\left.\frac{\partial p(T, \mu)}{\partial \mu}\right|_{T_{0}^{-}}=n_{-}=\left.\frac{\partial p(T, \mu)}{\partial \mu}\right|_{T_{0}^{+}} .
$$

This implies that even though there is a first order transition at $T_{0}$, the densities are equal. This is known in thermodynamics as a point of equal concentration. Since the transition is of first order, the entropies between the two phases differ at $T_{0}$.

We assume that the crossover line terminates at $T_{0}$, so in the chiral limit, $T_{0}$ coincides with the Lifshitz critical endpoint, $\tilde{\mathcal{C}}$. We cannot prove that $T_{0}$ is the shadow of $\tilde{\mathcal{C}}$, but it is a most natural conjecture.

It is clearly challenging to distinguish the fluctuations of a critical endpoint [5-10] from those of a Lifshitz regime. We leave this subject for future study. This is particularly true for heavy ion collisions, which are limited in both time and space.

In conclusion, it is surprising that there are such close analogies between the phase transitions in condensed matter systems, such as smectics and inhomogeneous polymers, and those of QCD. While our analysis is a first step, it may directly impact our understanding of the collisions of heavy ions at low energies.

\section{ACKNOWLEDGMENTS}

R. D. P. thanks G. Dunne for discussions on NJL models, the organizers of the Seventh International Conference on New Frontiers in Physics for the invitation to speak on this work, and K. Bugaev and K. Redlich for discussions at this meeting. R. D.P. is funded by the U.S. Department of Energy for support under Contract No. DE-SC0012704; A.M. T. is funded by Condensed Matter Physics and Materials Science Division, under the U.S. Department of Energy, Contract No. DE-SC0012704.
[1] J. B. Kogut and M. A. Stephanov, The phases of quantum chromodynamics: From confinement to extreme environments, Cambridge Monographs on Particle Physics, Nuclear Physics and Cosmology Vol. 21 (2003), pp. 1-9, https://doi.org/10.1017/CBO9780511534980;K. Yagi, T. Hatsuda, and Y. Miake, Quark-gluon plasma: From big bang to little bang, Camb. Monogr. Part. Phys. Nucl. Phys. Cosmol. 23, 1 (2005); K. Fukushima and T. Hatsuda, The phase diagram of dense QCD, Rep. Prog. Phys. 74, 014001 (2011).

[2] S. Borsanyi, Z. Fodor, C. Hoelbling, S. D. Katz, S. Krieg, and K. K. Szabo, Full result for the QCD equation of state with $2+1$ flavors, Phys. Lett. B 730, 99 (2014).

[3] A. Bazavov, T. Bhattacharya, C. DeTar, H.-T. Ding, S. Gottlieb, R. Gupta, P. Hegde, U. M. Heller, F. Karsch, E. Laermann, L. Levkova, S. Mukherjee, P. Petreczky, C. Schmidt, C. Schroeder, R. A. Soltz, W. Soeldner, R. Sugar, M. Wagner, and P. Vranas, The equation of state in $(2+1)$ flavor QCD, Phys. Rev. D 90, 094503 (2014).

[4] C. Ratti, Lattice QCD: Bulk and transport properties of QCD matter, Nucl. Phys. A956, 51 (2016).

[5] M. Asakawa and K. Yazaki, Chiral restoration at finite density and temperature, Nucl. Phys. A504, 668 (1989).

[6] M. A. Stephanov, K. Rajagopal, and E. V. Shuryak, Signatures of the Tricritical Point in QCD, Phys. Rev. Lett. 81, 4816 (1998).

[7] M. A. Stephanov, K. Rajagopal, and E. V. Shuryak, Eventby-event fluctuations in heavy ion collisions and the QCD critical point, Phys. Rev. D 60, 114028 (1999).

[8] D. T. Son and M. A. Stephanov, Dynamic universality class of the QCD critical point, Phys. Rev. D 70, 056001 (2004).
[9] Y. Hatta and T. Ikeda, Universality, the QCD critical/ tricritical point and the quark number susceptibility, Phys. Rev. D 67, 014028 (2003).

[10] M. A. Stephanov, Non-Gaussian Fluctuations Near the QCD Critical Point, Phys. Rev. Lett. 102, 032301 (2009).

[11] R. M. Hornreich, M. Luban, and S. Shtrikman, Critical Behavior at the Onset of $\boldsymbol{k}$-Space Instability on the $\lambda$ Line, Phys. Rev. Lett. 35, 1678 (1975).

[12] R. M. Hornreich, The Lifshitz point: Phase diagrams and critical behavior, J. Magn. Magn. Mater. 15, 387 (1980).

[13] P. M. Chaikin and T. C. Lubensky, Principles of Condensed Matter Physics (Cambridge University Press, Cambridge, England, 2010).

[14] H. W. Diehl, Critical behavior at M-axial Lifshitz points, Acta Phys. Slovaca 52, 271 (2002).

[15] A. Erzan and G. Stell, Isotropic Lifshitz point in $2<d<4$ dimensions, Phys. Rev. B 16, 4146 (1977).

[16] J. Sak and G. S. Grest, Critical exponents for the Lifshitz point: Epsilon expansion, Phys. Rev. B 17, 3602 (1978).

[17] G. S. Grest and J. Sak, Low-temperature renormalization group for the Lifshitz point, Phys. Rev. B 17, 3607 (1978).

[18] G. H. Fredrickson and F. S. Bates, Design of bicontinuous polymeric microemulsions, J. Polym. Sci. 35, 2775 (1997).

[19] D. Duchs, V. Genesan, G. H. Fredrickson, and F. Schmid, Fluctuation effects in ternary $\mathrm{AB}+\mathrm{A}+\mathrm{B}$ polymeric emulsions, Macromolecules 36, 9237 (2003).

[20] G. H. Fredrickson, The Equilibrium Theory of Inhomogeneous Polymers (Clarendon Press, Oxford, 2010).

[21] B. H. Jones and T. P. Lodge, Nanocasting nanoporous inorganic and organic materials from polymeric bicontinuous microemulsion templates, Polym. J. 44, 131 (2012). 
[22] M. E. Cates, Complex fluids: The physics of emulsions, arXiv:1209.2290.

[23] A. Bonanno and D. Zappala, Isotropic Lifshitz critical behavior from the functional renormalization group, Nucl. Phys. B893, 501 (2015).

[24] D. Zappala, Isotropic Lifshitz point in the $\mathrm{O}(\mathrm{N})$ Theory, Phys. Lett. B 773, 213 (2017).

[25] D. Zappala, Indications of isotropic Lifshitz points in four dimensions, Phys. Rev. D 98, 085005 (2018).

[26] A. W. Overhauser, Structure of Nuclear Matter, Phys. Rev. Lett. 4, 415 (1960).

[27] A. B. Migdal, Pion condensation Zh. Eksp. Teor. Fiz. 61, 2210 (1971) [Sov. Phys. JETP 36, 1052 (1973)].

[28] R. F. Sawyer, Condensed Pion Phase in Neutron Star Matter, Phys. Rev. Lett. 29, 382 (1972).

[29] D. J. Scalapino, Pion Condensate in Dense Nuclear Matter, Phys. Rev. Lett. 29, 386 (1972).

[30] R. F. Sawyer and D. J. Scalapino, Pion condensation in superdense nuclear matter, Phys. Rev. D 7, 953 (1973).

[31] A. B. Migdal, Pi Condensation in Nuclear Matter, Phys. Rev. Lett. 31, 257 (1973).

[32] A. B. Migdal, Pion fields in nuclear matter, Rev. Mod. Phys. 50, 107 (1978).

[33] A. B. Migdal, E. E. Saperstein, M. A. Troitsky, and D. N. Voskresensky, Pion degrees of freedom in nuclear matter, Phys. Rep. 192, 179 (1990).

[34] H. Kleinert, No pion condensate in nuclear matter due to fluctuations, Phys. Lett. 102B, 1 (1981).

[35] G. Baym, B. L. Friman, and G. Grinstein, Fluctuations and long range order in finite temperature pion condensates, Nucl. Phys. B210, 193 (1982).

[36] K. Kolehmainen and G. Baym, Pion condensation at finite temperature. 2. Simple models including thermal excitations of the pion field, Nucl. Phys. A382, 528 (1982).

[37] G. G. Bunatian and I. N. Mishustin, Thermodynamical theory of pion condensation, Nucl. Phys. A404, 525 (1983).

[38] T. Takatsuka and R. Tamagaki, $\pi^{0}$ condensation in dense symmetric nuclear matter at finite temperature, Prog. Theor. Phys. 77, 362 (1987).

[39] H. Kleinert and B. Van den Bossche, No massless pions in Nambu-Jona-Lasinio model due to chiral fluctuations, arXiv:hep-ph/9908284.

[40] D. B. Kaplan and A. E. Nelson, Strange goings on in dense nucleonic matter, Phys. Lett. B 175, 57 (1986).

[41] G. E. Brown, C.-H. Lee, M. Rho, and V. Thorsson, From kaon-nuclear interactions to kaon condensation, Nucl. Phys. A567, 937 (1994).

[42] G. E. Brown and M. Rho, From chiral mean field to Walecka mean field and kaon condensation, Nucl. Phys. A596, 503 (1996).

[43] G. E. Brown, C.-H. Lee, and Mannque Rho, Recent developments on kaon condensation and its astrophysical implications, Phys. Rep. 462, 1 (2008).

[44] V. Schon and M. Thies, Emergence of Skyrme crystal in Gross-Neveu and 't Hooft models at finite density, Phys. Rev. D 62, 096002 (2000).

[45] O. Schnetz, M. Thies, and K. Urlichs, Phase diagram of the Gross-Neveu model: Exact results and condensed matter precursors, Ann. Phys. (Amsterdam) 314, 425 (2004).
[46] M. Thies, From relativistic quantum fields to condensed matter and back again: Updating the Gross-Neveu phase diagram, J. Phys. A 39, 12707 (2006).

[47] G. Basar and G. V. Dunne, Self-Consistent Crystalline Condensate in Chiral Gross-Neveu and Bogoliubovde Gennes Systems, Phys. Rev. Lett. 100, 200404 (2008).

[48] G. Basar and G. V. Dunne, A twisted kink crystal in the chiral gross-Neveu model, Phys. Rev. D 78, 065022 (2008).

[49] G. Basar, G. V. Dunne, and M. Thies, Inhomogeneous condensates in the thermodynamics of the chiral NJL(2) model, Phys. Rev. D 79, 105012 (2009).

[50] P. Azaria, R. M. Konik, P. Lecheminant, T. Palmai, G. Takacs, and A. M. Tsvelik, Particle formation and ordering in strongly correlated fermionic systems: Solving a model of quantum chromodynamics, Phys. Rev. D 94, 045003 (2016).

[51] D. V. Deryagin, D. Y. Grigoriev, and V. A. Rubakov, Standing wave ground state in high density, zero temperature QCD at large N(c), Int. J. Mod. Phys. A 07, 659 (1992).

[52] E. Shuster and D. T. Son, On finite density QCD at large N(c), Nucl. Phys. B573, 434 (2000).

[53] B.-Y. Park, M. Rho, A. Wirzba, and I. Zahed, Dense QCD: Overhauser or BCS pairing?, Phys. Rev. D 62, 034015 (2000).

[54] R. Rapp, E. V. Shuryak, and I. Zahed, A chiral crystal in cold QCD matter at intermediate densities?, Phys. Rev. D 63, 034008 (2001).

[55] E. Nakano and T. Tatsumi, Chiral symmetry and density wave in quark matter, Phys. Rev. D 71, 114006 (2005).

[56] B. Bringoltz, Chiral crystals in strong-coupling lattice QCD at nonzero chemical potential, J. High Energy Phys. 03 (2007) 016.

[57] M. Sadzikowski, Comparison of the non-uniform chiral and 2SC phases at finite temperatures and densities, Phys. Lett. B 642, 238 (2006).

[58] B. Bringoltz, Volume dependence of two-dimensional large-N QCD with a nonzero density of baryons, Phys. Rev. D 79, 105021 (2009).

[59] K. Miura, T.Z. Nakano, and A. Ohnishi, Quarkyonic matter in lattice QCD at strong coupling, Prog. Theor. Phys. 122, 1045 (2009).

[60] B. Bringoltz, Solving two-dimensional large-N QCD with a nonzero density of baryons and arbitrary quark mass, Phys. Rev. D 79, 125006 (2009).

[61] S. Maedan, Influence of current mass on the spatially inhomogeneous chiral condensate, Prog. Theor. Phys. 123, 285 (2010).

[62] D. Nickel, How Many Phases Meet at the Chiral Critical Point?, Phys. Rev. Lett. 103, 072301 (2009).

[63] D. Nickel, Inhomogeneous phases in the Nambu-JonaLasino and quark-meson model, Phys. Rev. D 80, 074025 (2009).

[64] H. Abuki, G. Baym, T. Hatsuda, and N. Yamamoto, The NJL model of dense three-flavor matter with axial anomaly: the low temperature critical point and BEC-BCS diquark crossover, Phys. Rev. D 81, 125010 (2010). 
[65] S. Carignano, D. Nickel, and M. Buballa, Influence of vector interaction and Polyakov loop dynamics on inhomogeneous chiral symmetry breaking phases, Phys. Rev. D 82, 054009 (2010).

[66] T. L. Partyka and M. Sadzikowski, Chiral density waves in quarkyonic matter, Acta Phys. Pol. B 42, 1305 (2011).

[67] S. Carignano and M. Buballa, Two-dimensional chiral crystals in the NJL model, Phys. Rev. D 86, 074018 (2012).

[68] M. Buballa and S. Carignano, Self-bound quark matter in the NJL model revisited: From schematic droplets to domain-wall solitons, Phys. Rev. D 87, 054004 (2013).

[69] K. Kamikado, T. Kunihiro, K. Morita, and A. Ohnishi, Functional renormalization group study of phonon mode effects on chiral critical point, Prog. Theor. Exp. Phys. 2013, 053D01 (2013).

[70] H. Abuki, Ginzburg-Landau phase diagram of QCD near chiral critical point-chiral defect lattice and solitonic pion condensate, Phys. Lett. B 728, 427 (2014).

[71] B. Feng, E. J. Ferrer, and V. de la Incera, Quarkyonic chiral spirals in the Nambu-Jona-Lasinio approach, arXiv: 1304.0256.

[72] S. Karasawa and T. Tatsumi, Variational approach to the inhomogeneous chiral phase in quark matter, Phys. Rev. D 92, 116004 (2015).

[73] J. Moreira, B. Hiller, W. Broniowski, A. A. Osipov, and A.H. Blin, Nonuniform phases in a three-flavor Nambu-Jona-Lasinio model, Phys. Rev. D 89, 036009 (2014).

[74] D. Müller, M. Buballa, and J. Wambach, Dyson-Schwinger study of chiral density waves in QCD, Phys. Lett. B 727, 240 (2013).

[75] S. Carignano, M. Buballa, and B.-J. Schaefer, Inhomogeneous phases in the quark-meson model with vacuum fluctuations, Phys. Rev. D 90, 014033 (2014).

[76] T. Hayata and A. Yamamoto, Inhomogeneous Polyakov loop induced by inhomogeneous chiral condensates, Phys. Lett. B 744, 401 (2015).

[77] M. Kitazawa, T. Kunihiro, and Y. Nemoto, Emergence of soft quark excitations by the coupling with a soft mode of the QCD critical point, Phys. Rev. D 90, 116008 (2014).

[78] T. Kojo and G. Baym, Color screening in cold quark matter, Phys. Rev. D 89, 125008 (2014).

[79] T. Kojo, Chiral spirals from noncontinuous chiral symmetry: The gross-Neveu model results, Phys. Rev. D 90, 065030 (2014).

[80] J. Braun, F. Karbstein, S. Rechenberger, and D. Roscher, Crystalline ground states in Polyakov-loop extended Nambu-Jona-Lasinio models, Phys. Rev. D 93, 014032 (2016).

[81] M. Buballa and S. Carignano, Inhomogeneous chiral symmetry breaking in dense neutron-star matter, Eur. Phys. J. A 52, 57 (2016).

[82] S. Carignano, E. J. Ferrer, V. de la Incera, and L. Paulucci, Crystalline chiral condensates as a component of compact stars, Phys. Rev. D 92, 105018 (2015).
[83] J. P. Carlomagno, D. Gómez Dumm, and N. N. Scoccola, Inhomogeneous phases in nonlocal chiral quark models, Phys. Rev. D 92, 056007 (2015).

[84] M. Harada, H. K. Lee, Y.-L. Ma, and M. Rho, Inhomogeneous quark condensate in compressed Skyrmion matter, Phys. Rev. D 91, 096011 (2015).

[85] P. Adhikari and J. O. Andersen, Chiral density wave versus pion condensation in the $1+1$ dimensional NJL model, Phys. Rev. D 95, 054020 (2017).

[86] P. Adhikari and J. O. Andersen, Consistent regularization and renormalization in models with inhomogeneous phases, Phys. Rev. D 95, 036009 (2017).

[87] S. Carignano, M. Buballa, and W. Elkamhawy, Consistent parameter fixing in the quark-meson model with vacuum fluctuations, Phys. Rev. D 94, 034023 (2016).

[88] E. J. Ferrer and V. de la Incera, Exploring dense and cold QCD in magnetic fields, Eur. Phys. J. A 52, 266 (2016).

[89] S. Karasawa, T.-G. Lee, and T. Tatsumi, BrazovskiiDyugaev effect on the inhomogeneous chiral transition in quark matter, Prog. Theor. Exp. Phys. (2016), 043 D02.

[90] T. Yokota, T. Kunihiro, and K. Morita, Functional renormalization group analysis of the soft mode at the QCD critical point, Prog. Theor. Exp. Phys. (2016), 073D01.

[91] T. G. Khunjua, K. G. Klimenko, R. N. Zhokhov, and V. C. Zhukovsky, Inhomogeneous charged pion condensation in chiral asymmetric dense quark matter in the framework of $\mathrm{NJL}_{2}$ model, Phys. Rev. D 95, 105010 (2017).

[92] S. Carignano, F. Anzuini, O. Benhar, and M. Mannarelli, Crystalline phases by an improved gradient expansion technique, Phys. Rev. D 97, 036009 (2018).

[93] A. Heinz, F. Giacosa, and D. H. Rischke, Chiral density wave in nuclear matter, Nucl. Phys. A933, 34 (2015).

[94] A. Heinz, F. Giacosa, M. Wagner, and D. H. Rischke, Inhomogeneous condensation in effective models for QCD using the finite-mode approach, Phys. Rev. D 93, 014007 (2016).

[95] M. Buballa and S. Carignano, Inhomogeneous chiral condensates, Prog. Part. Nucl. Phys. 81, 39 (2015).

[96] T. Tatsumi and T. Muto, Quark beta decay in the inhomogeneous chiral phase and cooling of compact stars, Phys. Rev. D 89, 103005 (2014).

[97] T.-G. Lee, E. Nakano, Y. Tsue, T. Tatsumi, and B. Friman, Landau-Peierls instability in a Fulde-Ferrell type inhomogeneous chiral condensed phase, Phys. Rev. D 92, 034024 (2015).

[98] Y. Hidaka, K. Kamikado, T. Kanazawa, and T. Noumi, Phonons, pions and quasi-long-range order in spatially modulated chiral condensates, Phys. Rev. D 92, 034003 (2015).

[99] M. Nitta, S. Sasaki, and R. Yokokura, Spatially modulated vacua in relativistic field theories, Eur. Phys. J. C 78, 754 (2018).

[100] M. Nitta, S. Sasaki, and R. Yokokura, Supersymmetry breaking in spatially modulated vacua, Phys. Rev. D 96, 105022 (2017).

[101] R. Yoshiike, T.-G. Lee, and T. Tatsumi, Chiral pair fluctuations for the inhomogeneous chiral transition, Phys. Rev. D 95, 074010 (2017). 
[102] L. McLerran and R. D. Pisarski, Phases of cold, dense quarks at large N(c), Nucl. Phys. A796, 83 (2007).

[103] T. Kojo, Y. Hidaka, L. McLerran, and R. D. Pisarski, Quarkyonic chiral spirals, Nucl. Phys. A843, 37 (2010).

[104] T. Kojo, R. D. Pisarski, and A. M. Tsvelik, Covering the fermi surface with patches of quarkyonic chiral spirals, Phys. Rev. D 82, 074015 (2010).

[105] T. Kojo, Y. Hidaka, K. Fukushima, L. D. McLerran, and R. D. Pisarski, Interweaving chiral spirals, Nucl. Phys. A875, 94 (2012).

[106] A. Andronic et al., Hadron production in ultra-relativistic nuclear collisions: Quarkyonic matter and a triple point in the phase diagram of QCD, Nucl. Phys. A837, 65 (2010).

[107] S. A. Brazovskii, Phase transition of an isotropic system to a nonuniform state, Sov. Phys. JETP 41, 85 (1975) [Zh. Eksp. Teor. Fiz. 68, 175 (1975)].

[108] A. M. Dyugaev, Crystalline and liquid phases of a pion condensate, JETP Lett. 35, 420 (1982) [Pis'ma Zh. Eksp. Teor. Fiz. 35, 341 (1982)].

[109] P. C. Hohenberg and J. B. Swift, Metastability in fiuctuation-driven first-order transitions: Nucleation of lamellar phases, Phys. Rev. E 52, 1828 (1995).

[110] A. Andronic, An overview of the experimental study of quark-gluon matter in high-energy nucleus-nucleus collisions, Int. J. Mod. Phys. A 29, 1430047 (2014).

[111] A. Andronic, P. Braun-Munzinger, K. Redlich, and J. Stachel, Decoding the phase structure of QCD via particle production at high energy, Nature (London) 561, 321 (2018).

[112] L. Adamczyk et al. (STAR Collaboration), Bulk properties of the medium produced in relativistic heavy-ion collisions from the beam energy scan program, Phys. Rev. C 96, 044904 (2017).

[113] K. A. Bugaev, R. Emaus, V. V. Sagun, A. I. Ivanytskyi, L. V. Bravina, D. B. Blaschke, E. G. Nikonov, A. V. Taranenko, E. E. Zabrodin, and G. M. Zinovjev, Threshold collision energy of the QCD phase diagram tricritical endpoint, Phys. Part. Nucl. Lett. 15, 210 (2018).

[114] K. A. Bugaev, R. Emaus, V. V. Sagun, A. I. Ivanytskyi, L. V. Bravina, D. B. Blaschke, E. G. Nikonov, A. V. Taranenko, E. E. Zabrodin, and G. M. Zinovjev, Evidence of the QCD tricritical endpoint existence at NICA-FAIR energies, KnE Energ. Phys. 3, 313 (2018).

[115] K. A. Bugaev, A. I. Ivanytskyi, V. V. Sagun, G. M. Zinovjev, E. G. Nikonov, R. Emaus, L. V. Bravina, E. E. Zabrodin, and A. V. Taranenko, Probing the tricritical endpoint of QCD phase diagram at NICA-FAIR energies, EPJ Web Conf. 182, 02021 (2018).

[116] S. Jowzaee (STAR Collaboration), Rapidity correlations in the RHIC beam energy scan data, Nucl. Phys. A967, 792 (2017).

[117] X. Luo and N. Xu, Search for the QCD critical point with fluctuations of conserved quantities in relativistic heavyion collisions at RHIC: An overview, Nuclear Science and Techniques 28, 112 (2017).

[118] S. Hands, S. Kim, and J.-I. Skullerud, A quarkyonic phase in dense two color matter?, Phys. Rev. D 81, 091502 (2010).
[119] S. Hands, P. Kenny, S. Kim, and J.-I. Skullerud, Lattice study of dense matter with two colors and four flavors, Eur. Phys. J. A 47, 60 (2011).

[120] S. Cotter, P. Giudice, S. Hands, and J.-I. Skullerud, Towards the phase diagram of dense two-color matter, Phys. Rev. D 87, 034507 (2013).

[121] V. V. Braguta, E. M. Ilgenfritz, A. Yu. Kotov, B. Petersson, and S. A. Skinderev, Study of QCD phase diagram with non-zero chiral chemical potential, Phys. Rev. D 93, 034509 (2016).

[122] V. V. Braguta, E. M. Ilgenfritz, A. Yu. Kotov, A. V. Molochkov, and A. A. Nikolaev, Study of the phase diagram of dense two-color QCD within lattice simulation, Phys. Rev. D 94, 114510 (2016).

[123] V. G. Bornyakov, V. V. Braguta, E. M. Ilgenfritz, A. Yu. Kotov, A. V. Molochkov, and A. A. Nikolaev, Observation of deconfinement in a cold dense quark medium, J. High Energy Phys. 03 (2018) 161.

[124] A. Kurkela, E. S. Fraga, J. Schaffner-Bielich, and A. Vuorinen, Constraining neutron star matter with quantum chromodynamics, Astrophys. J. 789, 127 (2014).

[125] E. S. Fraga, A. Kurkela, and A. Vuorinen, Neutron star structure from QCD, Eur. Phys. J. A 52, 49 (2016).

[126] E. Braaten and A. Nieto, On the Convergence of Perturbative QCD at High Temperature, Phys. Rev. Lett. 76, 1417 (1996).

[127] E. Braaten and A. Nieto, Free energy of QCD at high temperature, Phys. Rev. D 53, 3421 (1996).

[128] M. Laine and Y. Schroder, Two-loop QCD gauge coupling at high temperatures, J. High Energy Phys. 03 (2005) 067.

[129] Y. Schroder and M. Laine, Spatial string tension revisited, Proc. Sci., LAT2005 (2006) 180.

[130] A. Kurkela and A. Vuorinen, Cool Quark Matter, Phys. Rev. Lett. 117, 042501 (2016).

[131] I. Ghisoiu, T. Gorda, A. Kurkela, P. Romatschke, M. Säppi, and A. Vuorinen, On high-order perturbative calculations at finite density, Nucl. Phys. B915, 102 (2017).

[132] R. F. Wagenbrunn and L. Ya. Glozman, Chiral symmetry patterns of excited mesons with the Coulomb-like linear confinement, Phys. Rev. D 75, 036007 (2007).

[133] L. Ya. Glozman and R. F. Wagenbrunn, Chirally symmetric but confining dense and cold matter, Phys. Rev. D 77, 054027 (2008).

[134] L. Ya. Glozman, $S U\left(2 N_{f}\right)$ hidden symmetry of QCD, arXiv:1511.05857.

[135] L. Ya. Glozman, $S U\left(2 N_{F}\right)$ symmetry of confinement in QCD and its observation at high temperature, EPJ Web Conf. 182, 02046 (2018).

[136] P. Di Francesco, P. Mathieu, and D. Senechal, Conformal Field Theory, Graduate Texts in Contemporary Physics (Springer-Verlag, New York, 1997).

[137] V. Skokov, A. Yu. Illarionov, and V. Toneev, Estimate of the magnetic field strength in heavy-ion collisions, Int. J. Mod. Phys. A 24, 5925 (2009).

[138] E. J. Ferrer, V. de la Incera, and A. Sanchez, Quarkyonic chiral spirals in a magnetic field, Acta Phys. Pol. B Proc. Suppl. 5, 679 (2012).

[139] T. Hayata, Y. Hidaka, and A. Yamamoto, Temporal chiral spiral in QCD in the presence of strong magnetic fields, Phys. Rev. D 89, 085011 (2014). 
[140] T. Tatsumi, K. Nishiyama, and S. Karasawa, Novel Lifshitz point for chiral transition in the magnetic field, Phys. Lett. B 743, 66 (2015).

[141] K. Nishiyama, S. Karasawa, and T. Tatsumi, Hybrid chiral condensate in the external magnetic field, Phys. Rev. D 92 , 036008 (2015).

[142] R. Yoshiike and T. Tatsumi, Symmetry breaking effect on the inhomogeneous chiral transition in the magnetic field, Phys. Rev. D 92, 116009 (2015).

[143] Y. Bu and S. Lin, Holographic magnetized chiral density wave, Chin. Phys. C 42, 114104 (2018).

[144] R. D. Pisarski, Critical line for H superfluidity in strange quark matter?, Phys. Rev. C 62, 035202 (2000).

[145] E. Witten, Global aspects of current algebra, Nucl. Phys. B223, 422 (1983).

[146] P. Horava, Spectral Dimension of the Universe in Quantum Gravity at a Lifshitz Point, Phys. Rev. Lett. 102, 161301 (2009).

[147] P. Horava, Quantum gravity at a Lifshitz point, Phys. Rev. D 79, 084008 (2009).

[148] S. Mukohyama, Horava-Lifshitz cosmology: A review, Classical Quantum Gravity 27, 223101 (2010).

[149] L. Sebastiani, S. Vagnozzi, and R. Myrzakulov, Mimetic gravity: A review of recent developments and applications to cosmology and astrophysics, Adv. High Energy Phys. 2017, 1 (2017).

[150] D. J. Amit, Field Theory, the Renormalization Group, and Critical Phenomena (World Scientific, Singapore, 2005).

[151] S. R. Coleman and E. J. Weinberg, Radiative corrections as the origin of spontaneous symmetry breaking, Phys. Rev. D 7, 1888 (1973).

[152] B. I. Halperin, T. C. Lubensky, and Shang-keng Ma, First Order Phase Transitions in Superconductors and Smectic a Liquid Crystals, Phys. Rev. Lett. 32, 292 (1974).

[153] P. Bak, S. Krinsky, and D. Mukamel, First-Order Transitions, Symmetry, and the $\epsilon$ Expansion, Phys. Rev. Lett. 36, 52 (1976).

[154] R. D. Pisarski and F. Wilczek, Remarks on the chiral phase transition in chromodynamics, Phys. Rev. D 29, 338 (1984).

[155] Y. Nakayama and T. Ohtsuki, Approaching the conformal window of $O(n) \times O(m)$ symmetric Landau-Ginzburg models using the conformal bootstrap, Phys. Rev. D 89, 126009 (2014).
[156] Y. Nakayama and T. Ohtsuki, Bootstrapping phase transitions in QCD and frustrated spin systems, Phys. Rev. D 91, 021901 (2015).

[157] M. Buballa and S. Carignano, Inhomogeneous chiral phases away from the chiral limit, Phys. Lett. B 791, 361 (2019).

[158] C. S. Fischer, QCD at finite temperature and chemical potential from Dyson-Schwinger equations, Prog. Part. Nucl. Phys. 105, 1 (2019).

[159] To one loop order, the simplest NJL model involves the determinant $\operatorname{tr} \log (\not \partial+\sigma)$. This is invariant under a uniform scaling of both $\not$ and $\sigma: \not \partial \rightarrow \kappa \not$ and $\sigma \rightarrow \kappa \sigma$. This implies that the coefficients of $\left(\partial_{\mu} \sigma\right)^{2}$ and $\sigma^{4}$ are equal, along with many others. These relations are no longer valid once more four fermion couplings are included. We thank G. Dunne for discussions on this point.

[160] X. An, D. Mesterházy, and M. A. Stephanov, On spinodal points and Lee-Yang edge singularities, J. Stat. Mech. (2018) 033207.

[161] R. Bellwied, S. Borsanyi, Z. Fodor, S. D. Katz, A. Pasztor, C. Ratti, and K. K. Szabo, Fluctuations and correlations in high temperature QCD, Phys. Rev. D 92, 114505 (2015).

[162] R. Bellwied, S. Borsanyi, Z. Fodor, J. Günther, S. D. Katz, C. Ratti, and K. K. Szabo, The QCD phase diagram from analytic continuation, Phys. Lett. B 751, 559 (2015).

[163] J. Gunther, R. Bellwied, S. Borsanyi, Z. Fodor, S. D. Katz, A. Pasztor, and C. Ratti, The QCD equation of state at finite density from analytical continuation, EPJ Web Conf. 137, 07008 (2017).

[164] A. Bazavov et al. (HotQCD Collaboration), Skewness and kurtosis of net baryon-number distributions at small values of the baryon chemical potential, Phys. Rev. D 96, 074510 (2017).

[165] A. Bazavov et al., The QCD equation of state to $\mathcal{O}\left(\mu_{B}^{6}\right)$ from lattice QCD, Phys. Rev. D 95, 054504 (2017).

[166] V. Vovchenko, J. Steinheimer, O. Philipsen, and H. Stoecker, Cluster expansion model for QCD Baryon number fluctuations: No phase transition at $\mu_{B} / T<\pi$, Phys. Rev. D 97, 114030 (2018).

[167] G. A. Almasi, B. Friman, K. Morita, P. M. Lo, and K. Redlich, Fourier coefficients of the net-baryon number density and chiral criticality, arXiv:1805.04441. 